\title{
Dual bronchodilation in COPD: lung function and patient-reported outcomes - a review
}

\author{
This article was published in the following Dove Press journal: \\ International Journal of COPD \\ 30 December 2016 \\ Number of times this article has been viewed
}

\author{
David Price ${ }^{1,2}$ \\ Anders $\varnothing_{\text {strem }}^{3}$ \\ Mike Thomas ${ }^{4}$ \\ Tobias Welte ${ }^{5}$ \\ 'Department of Primary Care \\ Respiratory Medicine, Division \\ of Applied Health Sciences, \\ University of Aberdeen, Aberdeen, \\ UK; ${ }^{2}$ Observational and Pragmatic \\ Research Institute, Singapore; \\ ${ }^{3}$ Gransdalen Legesenter, Oslo, \\ Norway; ${ }^{4}$ Department of Primary \\ Care Research, University of \\ Southampton, Southampton, \\ UK; ${ }^{5}$ Department of Pulmonary \\ Medicine, Hannover Medical School, \\ Hannover, Germany
}

\begin{abstract}
Several fixed-dose combinations (FDCs) of long-acting bronchodilators (a long-acting muscarinic antagonist [LAMA] plus a long-acting $\beta_{2}$-agonist [LABA]) are available for the treatment of COPD. Studies of these FDCs have demonstrated substantial improvements in lung function (forced expiratory volume in 1 second) in comparison with their respective constituent monocomponents. Improvements in patient-reported outcomes (PROs), such as symptoms and health status, as well as exacerbation rates, have been reported compared with a LABA or LAMA alone, but results are less consistent. The inconsistencies may in part be owing to differences in study design, methods used to assess study end points, and patient populations. Nevertheless, these observations tend to support an association between improvements in forced expiratory volume in 1 second and improvements in symptom-based outcomes. In order to assess the effects of FDCs on PROs and evaluate relationships between PROs and changes in lung function, we performed a systematic literature search of publications reporting randomized controlled trials of FDCs. Results of this literature search were independently assessed by two reviewers, with a third reviewer resolving any conflicting results. In total, 22 Phase III randomized controlled trials of FDC bronchodilators in COPD were identified, with an additional study including a post-literature search (ten for indacaterol-glycopyrronium once daily, eight for umeclidiniumvilanterol once daily, three for tiotropium-olodaterol once daily, and two for aclidiniumformoterol twice daily). Results from these studies demonstrated that the LAMA-LABA FDCs significantly improved lung function compared with their component monotherapies or other single-agent treatments. Furthermore, LABA-LAMA combinations also generally improved symptoms and health status versus monotherapies, although some discrepancies between lung function and PROs were observed. Overall, the safety profiles of the FDCs were similar to placebo. Further research is required to examine more closely any relationship between lung function and PROs in patients receiving LABA-LAMA combinations.
\end{abstract}

Keywords: chronic obstructive pulmonary disease, combination therapy, dyspnea, forced expiratory volume, health status, spirometry

\section{Introduction}

Appropriate pharmacological management of COPD involves treatment with inhaled bronchodilators to reduce airflow limitation and hyperinflation. Most patient groups identified by the Global Initiative for Chronic Obstructive Lung Disease (GOLD) strategy can be managed using long-acting inhaled bronchodilators (long-acting muscarinic antagonists [LAMAs] and long-acting $\beta_{2}$-agonists [LABAs]), with or without inhaled corticosteroids. ${ }^{1}$ Fixed dose combinations (FDCs) provide potent bronchodilation versus single agents, ${ }^{2}$ with some advantage in terms of convenience and simplicity compared with combinations administered via separate inhalers. Beta agonists (BAs) and muscarinic antagonists (MAs) target different pathways to promote smooth-muscle relaxation and 
inhibit pulmonary constriction. Combining bronchodilators with different modes of action appears to be additive, providing greater efficacy versus component monotherapies. ${ }^{3}$ Randomized controlled trials (RCTs) of LABA-LAMA combinations via separate inhalers have generally shown improved lung function versus component monotherapies..$^{4-12}$

COPD is characterized by persistent airflow limitation, with forced expiratory volume in 1 second $\left(\mathrm{FEV}_{1}\right)$ to forced vital capacity ratio and percentage predicted $\mathrm{FEV}_{1}$ widely used as pathophysiological markers. ${ }^{1}$ However, COPD is multidimensional, with pulmonary, extrapulmonary, and systemic effects. Outcomes in addition to $\mathrm{FEV}_{1}$ are needed to assess disease burden and treatment efficacy. ${ }^{13}$ Spirometry is central to COPD diagnosis, but does not measure COPD burden in terms of health status. ${ }^{14}$ Additionally, spirometry is not always performed, and symptoms and exacerbation history can play important roles in treatment initiation and management. ${ }^{15}$ It is therefore important that spirometry is accompanied by assessments using patient-reported outcome (PRO) measures, such as breathlessness, physical functioning, and health status. ${ }^{14}$ Minimal clinically important differences (MCIDs) for these assessments and other COPD outcomes have been reviewed by Jones et al. ${ }^{14}$ Although a few studies and reports have examined associations between improved lung function (mainly $\mathrm{FEV}_{1}$ ) and PROs in COPD, ${ }^{16-21}$ the relationship between these efficacy measures is often weak, particularly for LAMAs and LABA-LAMA combinations. Here, we examine the evidence for the use of FDC bronchodilators in COPD, assess effects on PROs, and evaluate relationships between PROs and changes in lung function.

\section{Materials and methods}

This systematic literature search (not registered) was performed in accordance with the general principles of the Preferred Reporting Items for Systematic Reviews and Meta-Analyses (PRISMA). ${ }^{22}$ The literature search identified primary, English-language, RCT publications of fixedcombination bronchodilators reporting treatment effects on lung function and/or PROs in comparison with placebo, bronchodilator monotherapy, or inhaled corticosteroidLABA combinations in patients with COPD (Table S1). Data sources included a ProQuest search of Biosis, Biosis previews, Embase and Medline databases (January 1, 2006 to July 31,2014 ), and abstracts from principal respiratory congresses (January 1, 2009 to May 20, 2015; Table S2). These selected search dates ensured that all relevant publications on fixed-combination bronchodilators were captured.
Following the publication-database searches and during preparation of this manuscript (August 2015 onward), additional relevant articles became available, and thus these were added to the literature-search results.

All search results were extracted and gathered by a single party. Titles and abstracts were then scrutinized in parallel by two independent reviewers, and papers were categorized as relevant (where both reviewers categorized a paper as "relevant"), not relevant (where both reviewers judged a paper as "not relevant"), or potentially relevant (where one reviewer judged a paper as "relevant" and the other judged the same paper as "not relevant"). Irrelevant publications/ studies comprised review papers, unapproved treatment doses, nonclinical trials, incorrect drug, or incorrect disease. Conflicting results were resolved by a third reviewer, who provided input as to whether the abstract was of potential relevance based on the same criteria as the first reviewers. To reduce the risk of omitting relevant studies/papers, all relevant and potentially relevant results were subsequently reviewed by the authors, who had the final decision regarding which publications to take to the next review level. Where relevance was not discernible from abstracts, full copies of author-confirmed relevant/potentially relevant articles were further assessed by two reviewers and conflicts resolved by a third reviewer. Data from the literature describing treatment differences with the FDC and comparator are summarized - according to end point - using least-squares mean (LSM) and 95\% confidence interval (95\% CI), odds ratio $(\mathrm{OR})$, rate ratio, or hazard ratio (HR).

\section{Results \\ Systematic literature-search results}

The searches yielded 729 records, from which 35 primary publications were relevant (Figure 1). Literature searches were supplemented with information from ClinicalTrials.gov, and author expertise/knowledge (eg, if authors were aware that important publications were missing from search results). ${ }^{23}$ Between the time of the predefined search end (July 2014 for published manuscripts and May 2015 for congress abstracts) and the drafting of this manuscript (August 2015 onwards), additional FDC studies were being published, and are thus included in this review. ${ }^{23-34}$

\section{Trials of fixed-dose dual-combination bronchodilators}

FDC bronchodilators approved or in advanced clinical development for COPD include: indacaterol-glycopyrronium once daily (OD; QVA149; Ultibro ${ }^{\circledR}$ Breezhaler $^{\circledR}$; Novartis 


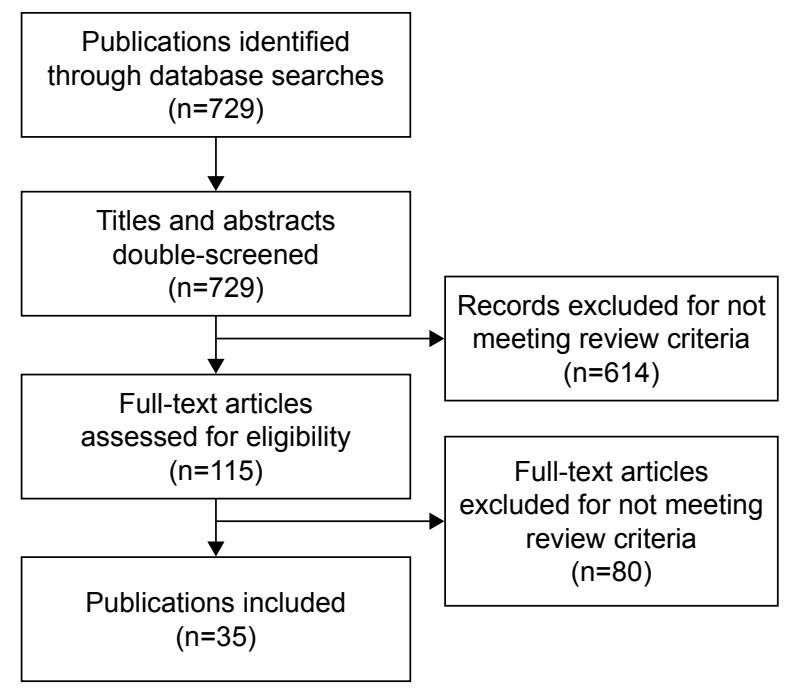

Figure I Flowchart of systematic literature search.

Notes: Reasons for exclusion comprised: not a primary publication or not containing novel data from clinical studies, non-COPD study, data not from a randomized clinical study, medication not combination LABA-LAMA bronchodilator treatment, and unapproved dose for a licensed combination therapy.

Abbreviations: LABA, long-acting $\beta_{2}$-agonist; LAMA, long-acting muscarinic antagonist.

International AG, Basel, Switzerland), umeclidiniumvilanterol 110/50 $\mu \mathrm{g}$ OD (Laventair/Anoro ${ }^{\circledR}$ Ellipta $^{\circledR}$; GlaxoSmithKline PLC, London, UK), tiotropium-olodaterol OD (Spiolto ${ }^{\circledR}$ Respimat $^{\circledR}$; Boehringer Ingelheim, Ingelheim, Germany), aclidinium-formoterol twice daily (bis in die [BID]; Duaklir ${ }^{\circledR}$ Genuair $^{\circledR}$; AstraZeneca PLC, London, UK) and glycopyrrolate-formoterol (PT003; AstraZeneca).

Indacaterol-glycopyrronium OD is approved in $>70$ countries. Of 13 large Phase III trials of indacaterol-glycopyrronium, publications are available for ten (SHINE, ILLUMINATE, BRIGHT, ENLIGHTEN, SPARK, BLAZE, BEACON, LANTERN, QUANTIFY, and FLAME), all of which report lung function and PRO data and are included in this review (Table 1). 2,24-26,35-40 These active-comparator and placebocontrolled trials ranged from 3 to 64 weeks in duration.

Umeclidinium-vilanterol 62.5/25 $\mu \mathrm{g}$ OD is approved in the US and EU (higher doses are not reviewed here). Findings from 12 Phase III trials had been reported in publications or conference abstracts at the time of the literature search, including: five 24-week studies, ${ }^{23,32,41}$ seven 12 -week studies, ${ }^{27,33,42,43}$ and one 52-week safety study $(125 / 25 \mu \mathrm{g}) .{ }^{45}$ Lung-function and PRO data have been fully reported for six of the eight trials listed in Table 1..$^{23,27,32,41}$

Tiotropium-olodaterol $(5 / 5 \mu \mathrm{g}$; lower doses are not reviewed) OD has been approved in more than 20 European countries, the US, Canada, and Australia since May 2015. Results from two 1-year studies with tiotropium-olodaterol
$5 / 5 \mu \mathrm{g}$ (included in this review; Table 1) have been reported and include data on lung function and health status versus the monocomponents. ${ }^{30}$ Results from an additional Phase III trial evaluating lung function and volume (VIVACITO) have been published (Table 1), ${ }^{31}$ two Phase III trials have been presented as abstracts, ${ }^{34,45}$ one further Phase III study has been completed (ClinicalTrials.gov NCT01536262) and four are ongoing (ClinicalTrials.gov NCT02006732, NCT01964352, NCT01969721, and NCT02085161).

Aclidinium-formoterol (400/12 $\mu \mathrm{g}$ BID) is approved in the EU. Findings from two of four Phase III trials have been fully reported comparing the combination therapy versus monocomponents or placebo, and are included in this paper (Table 1). ${ }^{28,29,46}$ Results from a 24-week Phase III study comparing aclidinium-formoterol with salmeterolfluticasone combination (SFC) BID had been published in abstract form at the time of the literature search. ${ }^{47}$ For glycopyrrolate-formoterol (in late-stage development), only Phase II congress abstracts are available. ${ }^{4-50}$ Three Phase III studies are ongoing (ClinicalTrials.gov NCT01854645, NCT01854658, and NCT01970878).

In this review, we focus on the 23 aforementioned published Phase III RCTs and listed in Table 1 (supplemented with results presented at major respiratory congresses, where applicable): ten with indacaterol-glycopyrronium OD, eight with umeclidinium-vilanterol OD, three with tiotropiumolodaterol OD, and two with aclidinium-formoterol BID. The remaining primary publications from the literature search were excluded, due to duplicate publications of the same results (eg, where a primary publication superseded several congress abstracts).

\section{Patient population and study design}

Patient populations, inclusion criteria, treatment blinding, and other characteristics differed between trials (Table 1). The majority of indacaterol-glycopyrronium OD studies enrolled symptomatic patients with moderate-to-severe airflow limitation (GOLD 2008, 2009, or 2010 classification), except for SPARK and FLAME, which enrolled patients with severe-to-very-severe or moderate-to-very-severe disease, respectively, and one or more exacerbations in the past year. ${ }^{2,24,26,35-40}$ The eight umeclidinium-vilanterol OD trials enrolled patients with moderate-to-severe or moderate-tovery-severe COPD who were symptomatic..$^{23,27,32,41}$ Patients in the tiotropium-olodaterol OD studies had moderate-tovery-severe COPD. ${ }^{30,31}$ The aclidinium-formoterol BID studies were conducted in patients with moderate-tosevere COPD. ${ }^{28,29}$ 


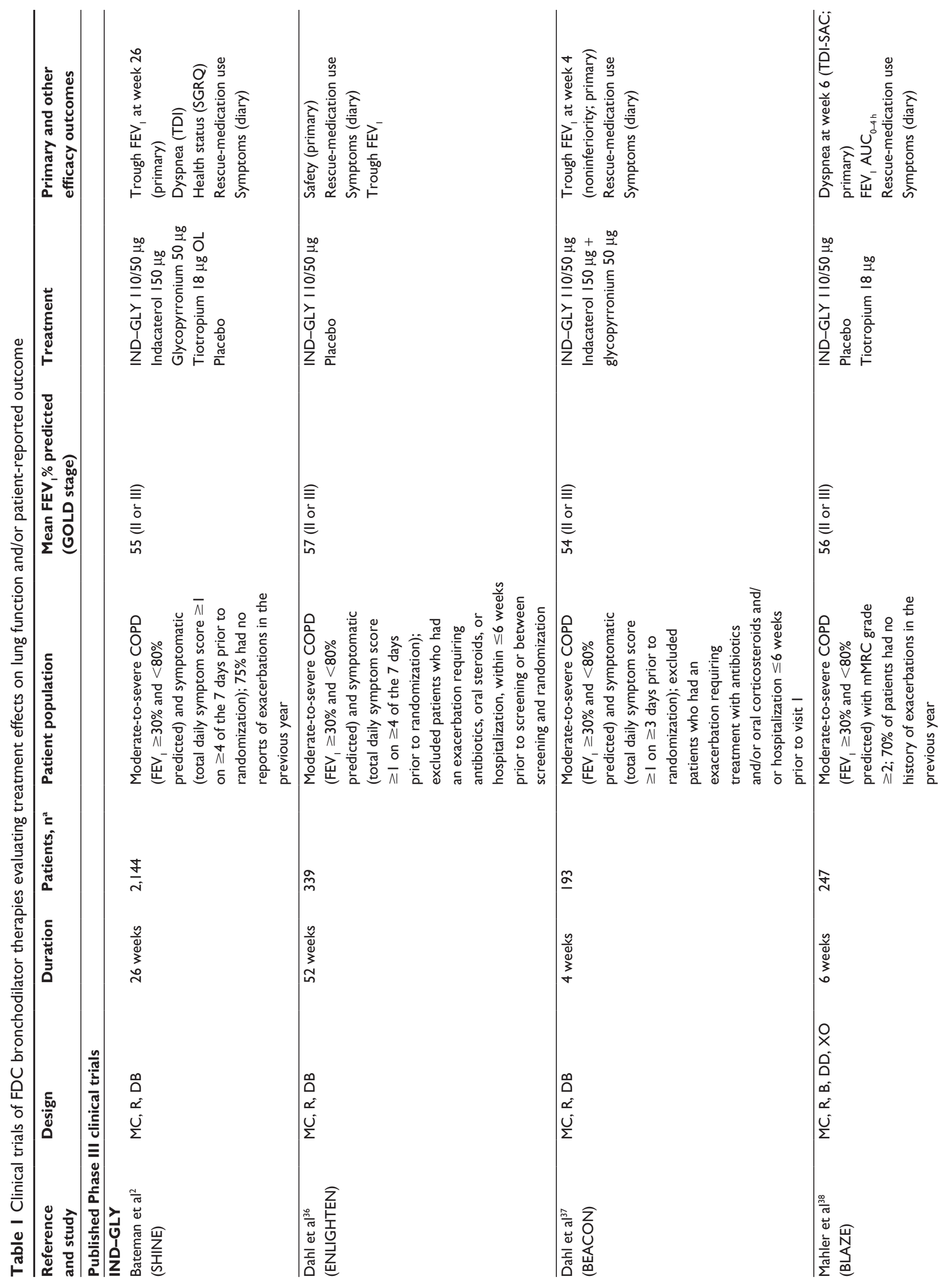




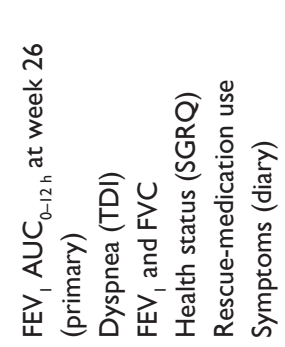

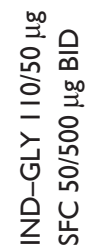

$$
\begin{aligned}
& \text { 辛 } \\
& \stackrel{0}{\equiv} \\
& \stackrel{0}{0}
\end{aligned}
$$

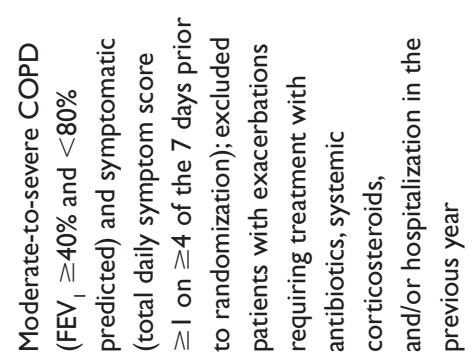

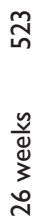

0
0
0
$\alpha$
$\alpha$
$\underline{u}$
$\Sigma$

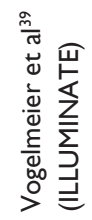

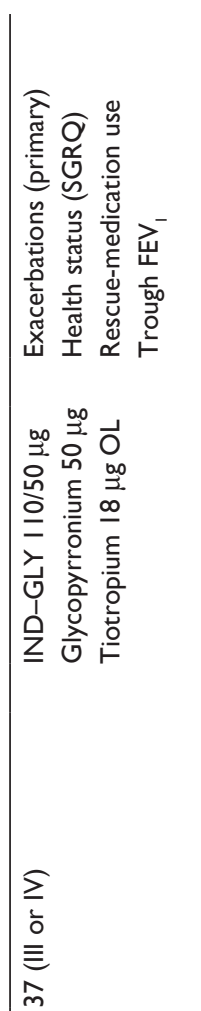
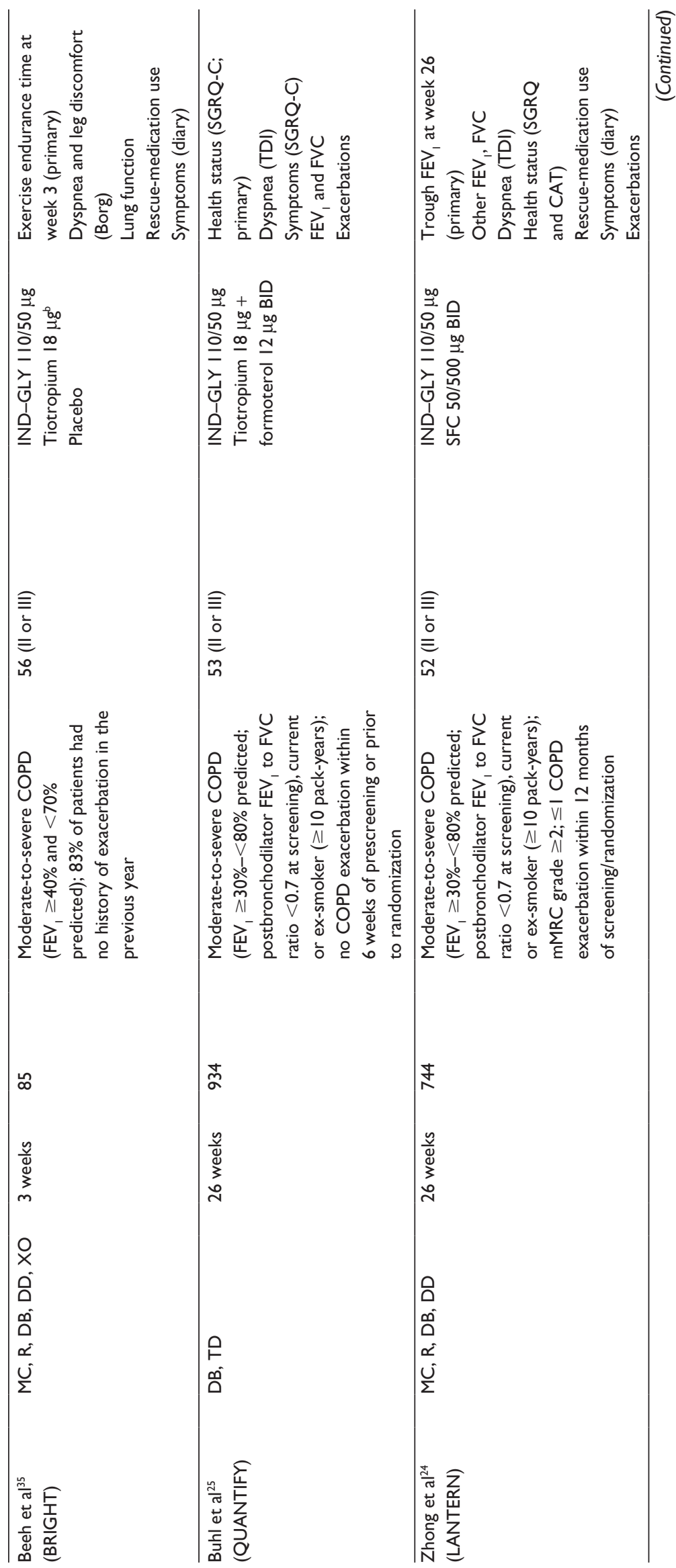


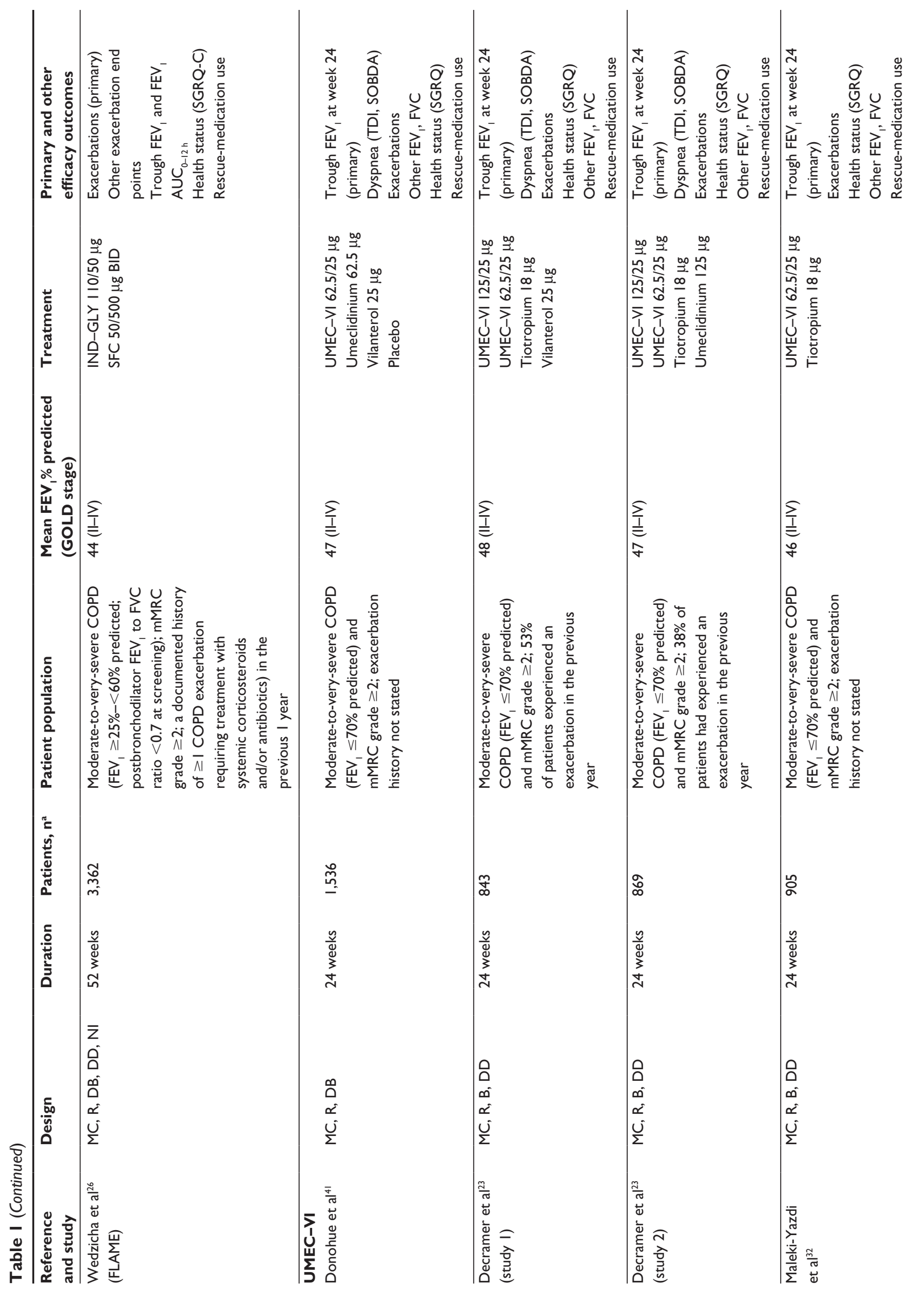




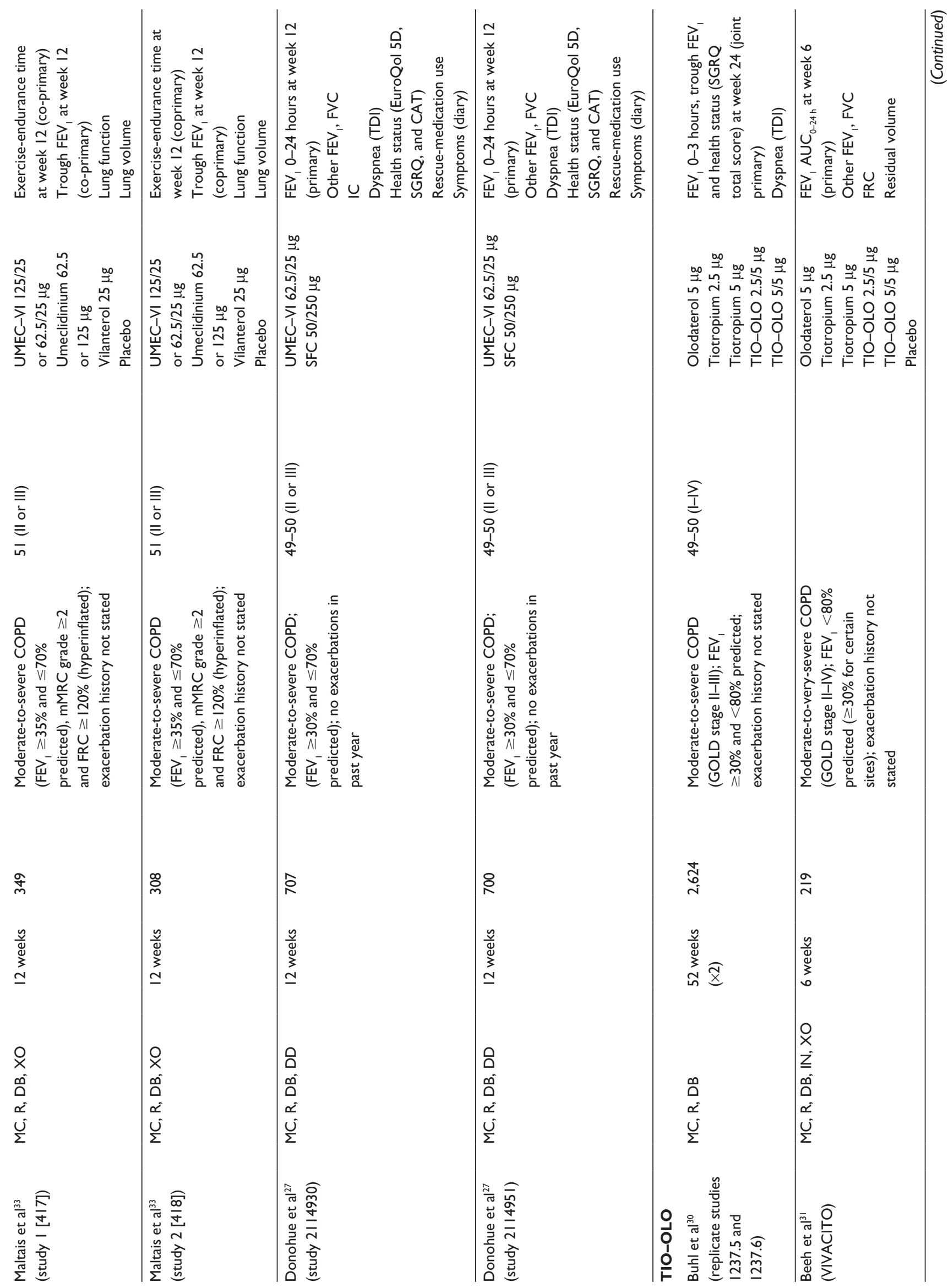




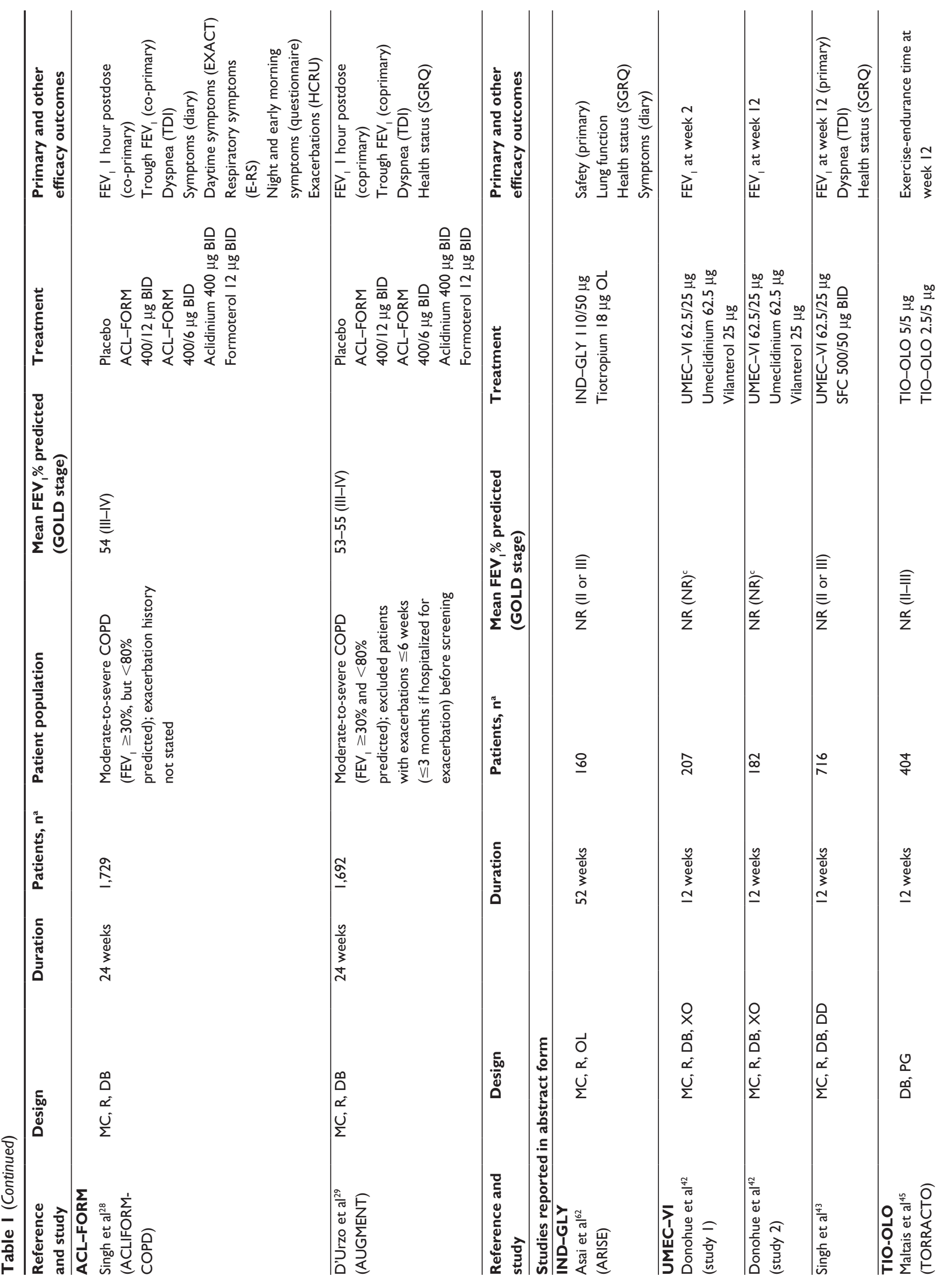




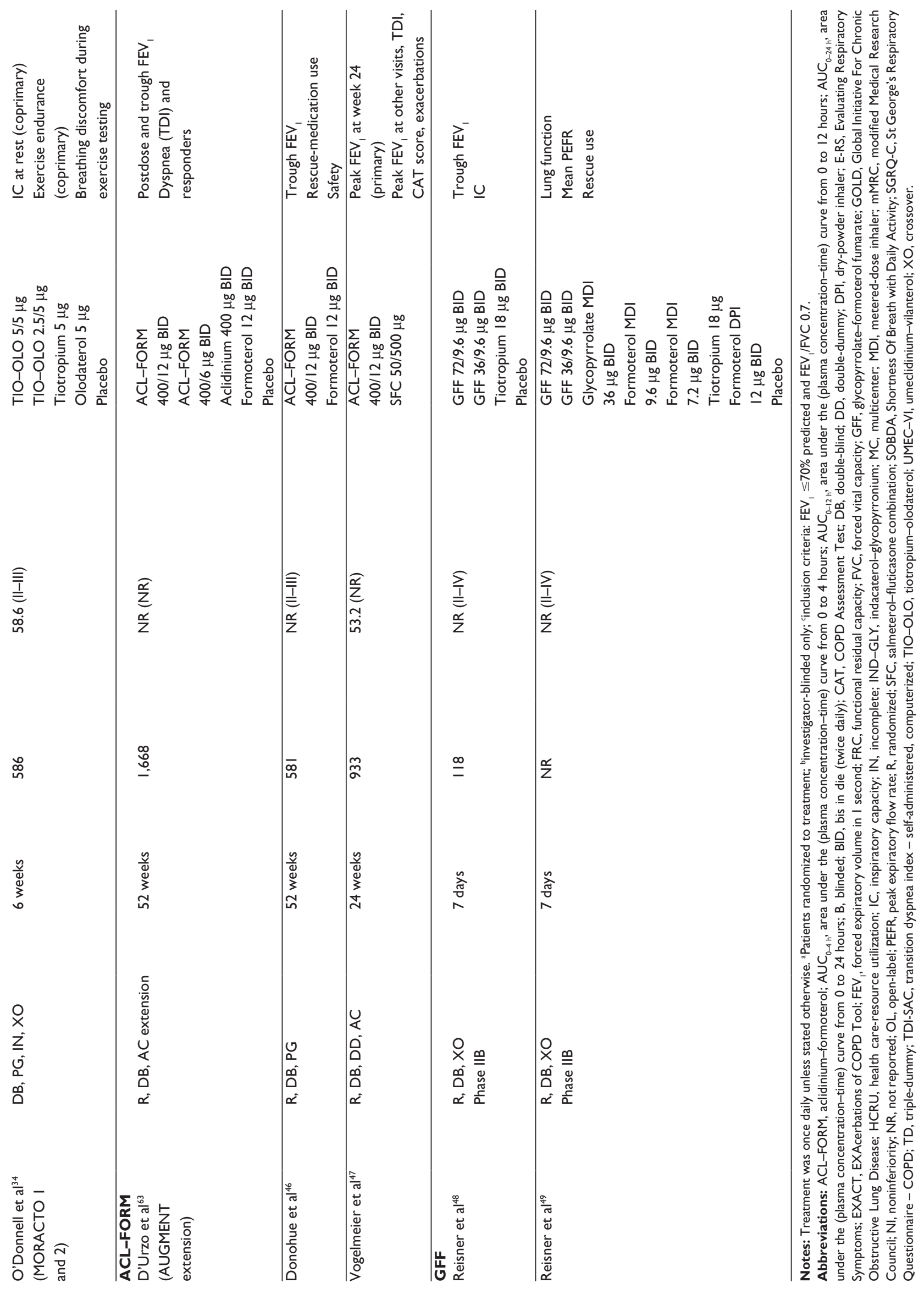




\section{Lung function}

Across eight trials (3-64 weeks), indacaterol-glycopyrronium OD provided significant LSM treatment differences in trough $\mathrm{FEV}_{1}$ of $60-80 \mathrm{~mL}$ versus tiotropium $18 \mu \mathrm{g}, 70-80 \mathrm{~mL}$ versus indacaterol $150 \mu \mathrm{g}$ or glycopyrronium $50 \mu \mathrm{g}$ alone, $68 \mathrm{~mL}$ versus tiotropium + formoterol $18 / 12 \mu \mathrm{g}, 62-72 \mathrm{~mL}$ versus SFC 50/500 $\mu \mathrm{g}$ BID, and $189-200 \mathrm{~mL}$ versus placebo (Table 2). ${ }^{2,24-26,35,36,39,40}$ Preliminary data suggest that the extent of $F E V_{1}$ improvement may vary: in a post hoc analysis of SHINE, data from patients in the spirometry subset who received indacaterol-glycopyrronium OD $(n=399)$ showed that $39.8 \%$ had an increase in $\mathrm{FEV}_{1}$ of $\geq 200 \mathrm{~mL}$ between baseline and week $26,23.8 \%$ achieved $\geq 300 \mathrm{~mL}$, and $13.1 \%$ had an increase of $\geq 400 \mathrm{~mL}^{.1}$

In three Phase III studies, LSM treatment differences in trough-FEV ${ }_{1}$ change from baseline to week 24 with umeclidinium-vilanterol $62.5 / 25 \mu \mathrm{g}$ OD were $60-112 \mathrm{~mL}$ versus tiotropium $18 \mu \mathrm{g}, 52 \mathrm{~mL}$ versus umeclidinium $62.5 \mu \mathrm{g}$, $22 \mathrm{~mL}$ versus umeclidinium $125 \mu \mathrm{g}$ (not statistically significant), 90-95 mL versus vilanterol $25 \mu \mathrm{g}$, and $167 \mathrm{~mL}$ versus placebo..$^{23,32,41}$ In two 12-week studies, umeclidiniumvilanterol $62.5 / 25 \mu \mathrm{g}$ produced greater increases in trough $\mathrm{FEV}_{1}$ versus individual components. ${ }^{33}$ In another two 12-week studies, umeclidinium-vilanterol $62.5 / 25 \mu \mathrm{g}$ resulted in

Table 2 Lung function: margin of efficacy of fixed combinations versus comparators in fully published studies

\begin{tabular}{|c|c|c|c|c|}
\hline Reference and study & Duration & Treatment & $\begin{array}{l}\text { Trough FEV, LSM } \\
(95 \% \mathrm{CI}) \text { treatment } \\
\text { difference at end point, } \mathrm{mL}\end{array}$ & $\begin{array}{l}\text { Other lung-function } \\
\text { parameters }\end{array}$ \\
\hline
\end{tabular}

\section{IND-GLY}

Bateman et al ${ }^{2}$ (SHINE) 26 weeks IND-GLY II0/50 $\mu \mathrm{g}$ OD vs

Indacaterol $150 \mu \mathrm{g}$ OD $\quad 70^{\mathrm{a}}(\mathrm{NR})$

Glycopyrronium $50 \mu \mathrm{g}$ OD $\quad 80^{\mathrm{a}}(\mathrm{NR})$

Tiotropium $18 \mu \mathrm{g}$ OD OL $\quad 70^{\mathrm{a}}(\mathrm{NR})$

Placebo 200 $(170-240)$

Dahl et $\mathrm{al}^{36}$ (ENLIGHTEN) 52 weeks IND-GLY I I0/50 $\mu \mathrm{g}$ OD

vs placebo $189^{a}(N R)$

IND-GLY provided significantly higher FEV, $\mathrm{AUC}_{0-4 \mathrm{~h}}$ and peak $\mathrm{FEV}_{\text {, compared }}$ with placebo, glycopyrronium, and tiotropium (all $P<0.0$ I)

$\mathrm{FEV}$, at 60 minutes postdose significantly greater with IND-GLY than placebo throughout the 52-week treatment period $(P<0.00 \mathrm{I}$ at all time points); IND-GLY improved FVC versus placebo over the 52-week treatment period $(P<0.001$ at all time points)

\begin{tabular}{|c|c|c|c|c|}
\hline Dahl et al ${ }^{37}$ (BEACON) & 4 weeks & $\begin{array}{l}\text { IND-GLY I I0/50 } \mu \mathrm{g} \text { OD vs } \\
\text { indacaterol I } 50 \mu \mathrm{g} \text { OD + } \\
\text { glycopyrronium } 50 \mu \mathrm{g} \text { OD }\end{array}$ & 5 (NR; NS for superiority) & $\begin{array}{l}\mathrm{FEV}_{1} \mathrm{AUC}_{0-4 \mathrm{~h}} \text { (day I and week 4) similar } \\
\text { between treatment groups }\end{array}$ \\
\hline Mahler et al ${ }^{38}$ (BLAZE) & 6 weeks & $\begin{array}{l}\text { IND-GLY I I 0/50 } \mu \mathrm{g} \text { OD vs } \\
\text { Placebo } \\
\text { Tiotropium I } 8 \mu \mathrm{g} \text { OD }\end{array}$ & $\begin{array}{l}330(0.31-0.36)^{\mathrm{a}, \mathrm{b}} \\
110(0.08-0.13)^{\mathrm{a}, \mathrm{b}}\end{array}$ & $\begin{array}{l}\mathrm{FEV}_{1} \mathrm{AUC}_{0-4 \mathrm{~h}} \text { postdose significantly higher } \\
\text { for IND-GLY vs tiotropium and placebo } \\
\text { at day I and week } 6 \text { (all } P<0.00 \text { I) }\end{array}$ \\
\hline $\begin{array}{l}\text { Vogelmeier et } \mathrm{a}^{39} \\
\text { (ILLUMINATE) }\end{array}$ & 26 weeks & $\begin{array}{l}\text { IND-GLY I I 0/50 } \mu \mathrm{g} \text { OD vs } \\
\text { SFC } 50 / 500 \mu \mathrm{g} \text { BID }\end{array}$ & $103^{\mathrm{a}}(65-14 \mid)$ & $\begin{array}{l}\text { Week } 26 \mathrm{FEV}_{1} \mathrm{AUC}_{0-12 \mathrm{~h}} \text { significantly } \\
\text { higher with IND-GLY than with SFC } \\
\text { (treatment difference I } 38 \mathrm{~mL}, 95 \% \\
\mathrm{Cl} 0.1-0.176 ; P<0.000 \text { I) }\end{array}$ \\
\hline Wedzicha et a ${ }^{40}$ (SPARK) & 64 weeks & $\begin{array}{l}\text { IND-GLY I I0/50 } \mu \mathrm{g} \text { OD vs } \\
\text { Glycopyrronium } 50 \mu \mathrm{g} \text { OD } \\
\text { Tiotropium } 18 \mu \mathrm{g} \text { OD OL }\end{array}$ & $\begin{array}{l}\text { Weeks 4-64: } \\
70-80^{\mathrm{a}} \text { (NR) } \\
60-80^{\mathrm{a}} \text { (NR) }\end{array}$ & NR \\
\hline 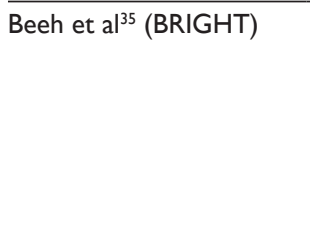 & 3 weeks & $\begin{array}{l}\text { IND-GLY I I0/50 } \mu \mathrm{g} \text { OD vs } \\
\text { Tiotropium } 18 \mu \mathrm{g} \mathrm{OD}^{\mathrm{a}} \\
\text { Placebo }\end{array}$ & $\begin{array}{l}100^{\mathrm{a}}(50-150) \\
200^{\mathrm{a}}(150-260)\end{array}$ & $\begin{array}{l}\text { At day } 2 \mathrm{I} \text {, mean treatment differences } \\
\text { in trough IC, FEV }{ }_{1} \text {, and FVC significantly } \\
\text { higher for IND-GLY vs placebo ( } 190 \text {, } \\
200 \text {, and } 280 \mathrm{~mL} \text {, respectively) and } \\
\text { vs tiotropium (I50, } 100 \text {, and II } 10 \mathrm{~mL} \text {, } \\
\text { respectively) }\end{array}$ \\
\hline Buhl et $\mathrm{a}^{25}$ (QUANTIFY) & 26 weeks & $\begin{array}{l}\text { IND/GLY I I0/50 } \mu \mathrm{g} \text { OD vs } \\
\text { tiotropium I } \mu \mathrm{g} \text { OD + } \\
\text { formoterol I } 2 \mu \mathrm{g} \mathrm{BID}\end{array}$ & $68^{a}(37-100)$ & $\begin{array}{l}\text { IND-GLY increased predose FVC vs } \\
\text { tiotropium + formoterol at week } 26 \\
(74 \mathrm{~mL}, 95 \% \mathrm{Cl}: 24-125 \mathrm{~mL} ; P=0.004)\end{array}$ \\
\hline
\end{tabular}

(Continued) 
Table 2 (Continued)

\begin{tabular}{|c|c|c|c|c|}
\hline Reference and study & Duration & Treatment & $\begin{array}{l}\text { Trough FEV, LSM } \\
(95 \% \mathrm{Cl}) \text { treatment } \\
\text { difference at end point, } \mathrm{mL}\end{array}$ & $\begin{array}{l}\text { Other lung-function } \\
\text { parameters }\end{array}$ \\
\hline $\begin{array}{l}\text { Zhong et a }{ }^{24} \\
\text { (LANTERN) }\end{array}$ & 26 weeks & $\begin{array}{l}\text { IND-GLY I I 0/50 } \mu \mathrm{g} \text { OD vs } \\
\text { SFC } 50 / 500 \mu \mathrm{g} \text { BID }\end{array}$ & $72^{\mathrm{a}}(40-104)$ & $\begin{array}{l}\text { Improvements in trough FEV, with } \\
\text { IND-GLY vs SFC observed at day I } \\
(\Delta=43 \mathrm{~mL}) \text { and reaching steady state } \\
\text { by week I } 2(\Delta=78 \mathrm{~mL} \text {, both } P<0.00 \mathrm{I}) \text {. } \\
\text { Improvements in FEV } \text { AUC }_{0-4 \mathrm{~h}} \text { at day I/ } \\
\text { week } 26 \text { with IND-GLY vs SFC } \\
(\Delta=65 / / 22 \mathrm{~mL} \text {, respectively). Peak FEV } \\
\text { higher at day I/week } 26 \text { with IND-GLY } \\
\text { vs SFC ( } P<0.00 \mathrm{I}) \text {. Trough FVC higher } \\
\text { for IND-GLY vs SFC ( } P<0.00 \mathrm{I}) \text {. } \\
\text { Improvements in peak FVC (over the } \\
\text { first } 4 \text { hours) with IND-GLY vs SFC at } \\
\text { day I/week } 26 \text { (all } P<0.00 \mathrm{I} \text { ) }\end{array}$ \\
\hline $\begin{array}{l}\text { Wedzicha et al }{ }^{26} \\
\text { (FLAME) }\end{array}$ & 52 weeks & $\begin{array}{l}\text { IND-GLY I I 0/50 } \mu \mathrm{g} \text { OD vs } \\
\text { SFC 50/500 } \mu \mathrm{g} \text { BID }\end{array}$ & $62^{\mathrm{a}}(\mathrm{NR})$ & $\begin{array}{l}\text { Change from baseline in } \mathrm{FEV}, \mathrm{AUC}_{0-12 \mathrm{~h}} \\
\text { (measured in a subgroup of } 556 \\
\text { patients) was significantly greater with } \\
\text { IND-GLY vs SFC at week } 52 \\
(\Delta=\mathrm{II} \mathrm{I} \mathrm{mL}, P<0.00 \mathrm{I})\end{array}$ \\
\hline
\end{tabular}

\section{UMEC-VI}

Donohue et $\mathrm{al}^{41}$
24 weeks

62.5/25 $\mu \mathrm{g}$ OD vs

Umeclidinium $62.5 \mu \mathrm{g}$ OD

Vilanterol $25 \mu \mathrm{g} O D$

Placebo

\begin{tabular}{|c|c|c|c|}
\hline \multirow[t]{4}{*}{ Decramer et a ${ }^{23}$ (study I) } & 24 weeks & UMEC-VI I $25 / 25 \mu \mathrm{g} \mathrm{OD}^{\mathrm{c}}$ & Change from baseline: \\
\hline & & UMEC-VI $62.5 / 25 \mu \mathrm{g}$ OD vs & \\
\hline & & Tiotropium I8 $\mu \mathrm{g}$ OD & $90^{\mathrm{a}}(39-141)$ \\
\hline & & Vilanterol $25 \mu \mathrm{g}$ OD & $90^{\mathrm{a}}(39-142)$ \\
\hline \multirow[t]{4}{*}{ Decramer et a ${ }^{23}$ (study 2$)$} & 24 weeks & UMEC-VI I $25 / 25 \mu \mathrm{g} \mathrm{OD}^{\mathrm{c}}$ & Change from baseline: \\
\hline & & UMEC-VI $62.5 / 25 \mu \mathrm{g}$ OD vs & \\
\hline & & Tiotropium $18 \mu \mathrm{g}$ OD & $60^{a}(10-109)$ \\
\hline & & Umeclidinium $125 \mu \mathrm{g}$ OD & 22 (-27 to 72$)$ \\
\hline$\overline{\text { Maleki-Yazdi et al }^{32}}$ & 24 weeks & $\begin{array}{l}\text { UMEC-VI } 62.5 / 25 \mu \mathrm{g} \text { OD vs } \\
\text { tiotropium } 18 \mu \mathrm{g} \text { OD }\end{array}$ & $\begin{array}{l}\text { Change from baseline: } \\
112^{\mathrm{a}}(8 \mid-144)\end{array}$ \\
\hline
\end{tabular}

Maltais et $\mathrm{al}^{33}$ (study 4I7) $\quad 12$ weeks $\quad$ UMEC-VI I25/25 $\mu \mathrm{g}$ OD $^{c} \quad$ Change from baseline vs

UMEC-VI 62.5/25 $\mu \mathrm{g}$ OD $\quad 2 \mathrm{I}^{\mathrm{a}}($ (172-249)

Umeclidinium $62.5 \mu \mathrm{g}$ OD $\quad 87^{\mathrm{a}}(30-143)$

Umeclidinium I $25 \mu \mathrm{g}$ OD $\quad 140^{\mathrm{a}}(84-96)$

Vilanterol $25 \mu \mathrm{g}$ OD

Maltais et al ${ }^{33}$ (study 4I8) 12 weeks UMEC-VI I25/25 $\mu \mathrm{g} \mathrm{OD}^{c}$

UMEC-VI 62.5/25 $\mu \mathrm{g}$ OD

Umeclidinium $62.5 \mu \mathrm{g}$ OD

Umeclidinium $125 \mu \mathrm{g}$ OD

Vilanterol $25 \mu \mathrm{g}$ OD

Donohue et $\mathrm{a}^{27}$

(study 2114930)

$\begin{array}{lll}12 \text { weeks } & \text { UMEC-VI 62.5/25 } \mu \mathrm{g} \text { OD vs } & \text { Change from baseline: } \\ & \text { SFC 50/250 } \mu \mathrm{g} \mathrm{BID} & 74^{\mathrm{a}}(38-110)\end{array}$
Change from baseline:

$52^{\mathrm{a}}(17-87)$

$95^{\mathrm{a}}(60-130)$

$167^{\text {a }}(128-207)$ placebo:

$99^{a}(50-148)$

Change from baseline vs Trough FEV, improved with UMEC-VI placebo:

$243^{\mathrm{a}}(202-284)$

$144^{\mathrm{a}}(86-203)$

$255^{\mathrm{a}}(193-318)$

$112^{a}(61-163)$

Improvements in trough FVC change from baseline observed at day 169 for UMEC-VI 62.5/25 $\mu \mathrm{g}$, UMEC $62.5 \mu \mathrm{g}$, and VI $25 \mu \mathrm{g}$ vs placebo $(248 \mathrm{~mL}, 175 \mathrm{~mL}$, and $105 \mathrm{~mL}$; all $P<0.002$ )

Mean 0- to 6-hour FEV, on day 168 for UMEC-VI (both doses) significantly improved vs tiotropium $18 \mu \mathrm{g}$

Mean 0- to 6-hour FEV, on day 168 for both doses of UMEC/VI improved vs tiotropium $18 \mu \mathrm{g}$ (nominal $P$-values)

Weighted mean FEV, over 0-6 hours postdose at day 168 improved for UMEC-VI vs tiotropium ( $105 \mathrm{~mL}, 95 \% \mathrm{Cl}$ 0.07 I-0.14; $P<0.00$ I)

Trough $F E V$, numerically improved with UMEC-VI I25/25 $\mu \mathrm{g}$ and UMEC-VI $62.5 / 25 \mu \mathrm{g}$ compared with placebo from day 2 to week 12 I25/25 $\mu \mathrm{g}$ and UMEC-VI 62.5/25 $\mu \mathrm{g}$ compared with placebo $(P<0.00 \mathrm{I})$ from day 2 to week 12

FEV, significantly improved for UMEC-VI vs SFC at all time points on day 84 (except 18 hours); significantly greater improvement in LSM trough FEV, from baseline for UMEC-VI vs SFC on day 85 (treatment difference $82 \mathrm{~mL}, P<0.00 \mathrm{I}$ ) 
Table 2 (Continued)

\begin{tabular}{|c|c|c|c|c|}
\hline Reference and study & Duration & Treatment & $\begin{array}{l}\text { Trough FEV, LSM } \\
(95 \% \mathrm{CI}) \text { treatment } \\
\text { difference at end point, } \mathrm{mL}\end{array}$ & Other lung-function parameters \\
\hline $\begin{array}{l}\text { Donohue et al }{ }^{27} \text { (study } \\
2 \text { II } 495 \text { I) }\end{array}$ & 12 weeks & $\begin{array}{l}\text { UMEC-VI } 62.5 / 25 \mu \mathrm{g} \text { OD vs } \\
\text { SFC } 50 / 250 \mu \mathrm{g} \text { BID }\end{array}$ & $\begin{array}{l}\text { Change from baseline: } \\
10 I^{\mathrm{a}}(63-139)\end{array}$ & $\begin{array}{l}\text { FEV, significantly improved for UMEC-VI } \\
\text { vs SFC at all time points on day } 84 \text {; } \\
\text { significantly greater improvement in LSM } \\
\text { trough FEV, from baseline for UMEC-VI } \\
\text { vs SFC on day } 85 \text { (treatment difference } \\
98 \mathrm{~mL}, P<0.00 \mathrm{I} \text { ) }\end{array}$ \\
\hline
\end{tabular}

Change from baseline at week 24:

Olodaterol $5 \mu \mathrm{g}$ OD $\quad 82^{\mathrm{a}}(59-106)$

Tiotropium $5 \mu \mathrm{g}$ OD $\quad$ 7 Ia $^{\mathrm{a}}(47-94)$

Tiotropium $2.5 \mu \mathrm{g}$ OD NR

Buhl et al ${ }^{30}$ (study 1237.6) 52 weeks $\quad$ TIO-OLO 2.5/5 $\mu \mathrm{g} \mathrm{OD}^{\mathrm{c}}$ TIO-OLO $5 / 5 \mu \mathrm{g}$ OD vs

Olodaterol $5 \mu \mathrm{g} O D$

Tiotropium $5 \mu \mathrm{g}$ OD

Tiotropium $2.5 \mu \mathrm{g}$ OD

Change from baseline

at week 24:

$88^{\mathrm{a}}(63-113)$

$50^{\mathrm{a}}(24-75)$

NR

\begin{tabular}{|c|c|c|c|}
\hline \multirow[t]{6}{*}{ Beeh et $\mathrm{a}^{31}$ (VIVACITO) } & \multirow[t]{6}{*}{6 weeks } & TIO-OLO $2.5 / 5 \mu \mathrm{g} \mathrm{OD}^{\mathrm{c}}$ & Adjusted mean difference: \\
\hline & & TIO-OLO $5 / 5 \mu \mathrm{g}$ OD vs & \\
\hline & & Olodaterol $5 \mu \mathrm{g}$ OD & $92^{\mathrm{a}}(\mathrm{NR})$ \\
\hline & & Tiotropium $5 \mu \mathrm{g}$ OD & $79^{a}(N R)$ \\
\hline & & Tiotropium $2.5 \mu \mathrm{g}$ OD & NR \\
\hline & & Placebo & $207^{\mathrm{a}}$ (NR) \\
\hline
\end{tabular}

\section{ACL-FORM}

Singh et a ${ }^{28}$ (ACLIFORM- 24 weeks COPD)

\begin{tabular}{|c|c|c|c|}
\hline & & \multirow{2}{*}{ Formoterol I $2 \mu \mathrm{g}$ BID } & \multirow{2}{*}{$85^{\mathrm{a}}$} \\
\hline & & & \\
\hline & & Aclidinium $400 \mu \mathrm{g}$ BID & $\sim 25^{d}$ \\
\hline & & Placebo & $143^{\mathrm{a}}$ \\
\hline \multirow{6}{*}{$\begin{array}{l}\text { D'Urzo et al }{ }^{29} \\
\text { (AUGMENT) }\end{array}$} & 24 weeks & ACL-FORM $400 / 6 \mu \mathrm{g}$ BID $^{c}$ & Change from baseline \\
\hline & & ACL-FORM 400/I2 $\mu \mathrm{g}$ & at week 24: \\
\hline & & BID vs & \\
\hline & & Formoterol $12 \mu \mathrm{g}$ BID & $45^{\mathrm{a}}$ \\
\hline & & Aclidinium $400 \mu \mathrm{g}$ BID & 28 \\
\hline & & Placebo & $129^{a}$ \\
\hline
\end{tabular}

Improvements observed for FEV, values on all test days over each of the 52-week studies; responses in trough FVC and FVC $\mathrm{AUC}_{0-3 \mathrm{~h}}$ over 24 weeks consistent with the primary end points

Improvements observed for $\mathrm{FEV}_{\text {, values }}$ on all test days over each of the 52-week studies; responses in trough FVC and FVC $\mathrm{AUC}_{0-3 \mathrm{~h}}$ over 24 weeks consistent with the primary end points

Significant improvement in $\mathrm{FEV}, \mathrm{AUC}_{0-24 \mathrm{~h}}$ and greater improvement in 24-hour

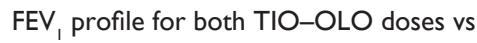
placebo and monotherapies at 6 weeks; similar pattern of response for FVC, FRC, and residual volume

Fast onset of action of both ACL-FORM doses on day I, with significant improvements in bronchodilation vs placebo at 5 minutes postdose

ACL-FORM (both doses) associated with significant changes from baseline in peak FEV , at day I and week 24 $(P<0.000$ I all comparisons); rapid bronchodilation occurred with significant $\mathrm{FEV}$, improvements 5 minutes postdose $(P<0.000 \mathrm{I})$

Notes: Treatment once daily unless stated otherwise. ${ }^{a}$ Significant treatment difference; ${ }^{\text {FFEV }}{ }_{1} \mathrm{AUC}_{0-4}$; ; dose not approved for use (ACL-FORM, dose not approved in EU); destimated from figure.

Abbreviations: $\mathrm{ACL}-\mathrm{FORM}$, aclidinium-formoterol; $\mathrm{AUC}_{0-3 \mathrm{~h}}$, area under the plasma concentration-time curve from 0 to 3 hours; $A \cup \mathrm{C}_{0-4 \mathrm{~h}}$, area under the (plasma concentration-time) curve from 0 to 4 hours; $\mathrm{AUC}_{0-12 \mathrm{~h}}$, area under the (plasma concentration-time) curve from 0 to 12 hours; $A \cup C_{0-24} \mathrm{~h}$, area under the (plasma concentration-time) curve from 0 to 24 hours; BID, bis in die (twice daily); Cl, confidence interval; $\mathrm{FEV}_{1}$, forced expiratory volume in I second; FVC, forced vital capacity; FRC, functional residual capacity; IC, inspiratory capacity; IND-GLY, indacaterol-glycopyrronium; LSM, least-squares mean; NR, not reported; NS, not significant; OD, once daily; OL, open-label; SFC, salmeterol/fluticasone combination; TIO-OLO, tiotropium-olodaterol; UMEC-VI, umeclidinium-vilanterol.

significant improvements in $\mathrm{FEV}_{1} 0-24$ hours and trough $\mathrm{FEV}_{1}$ compared with 50/250 $\mu \mathrm{g}$ BID. ${ }^{27}$

At week 24 of the two 1-year studies, tiotropiumolodaterol $5 / 5 \mu \mathrm{g}$ OD increased trough $\mathrm{FEV}_{1}$ by $82-88 \mathrm{~mL}$ versus olodaterol $5 \mu \mathrm{g}$ and by $50-71 \mathrm{~mL}$ versus tiotropium $5 \mu \mathrm{g} .{ }^{30}$ A 6-week incomplete crossover study showed improvements in 24-hour lung function with tiotropiumolodaterol $5 / 5 \mu \mathrm{g}$ versus components or placebo. ${ }^{31}$
Aclidinium-formoterol (400/12 $\mu \mathrm{g}$ BID) increased week 24 trough $\mathrm{FEV}_{1}$ significantly versus placebo $(143 \mathrm{~mL})$ and formoterol $(85 \mathrm{~mL})$ in the ACLIFORM study, but the smaller difference $(\sim 25 \mathrm{~mL})$ versus aclidinium BID was not statistically significant. ${ }^{28}$ Similar results were observed in the AUGMENT trial, with a significant difference for the combination versus formoterol $(45 \mathrm{~mL})$, but not aclidinium $(28 \mathrm{~mL}) .{ }^{29}$ 


\section{Symptoms}

Improvements in dyspnea and other symptoms were seen with fixed-dose LABA-LAMA therapies versus monotherapies and for indacaterol-glycopyrronium OD versus SFC BID. (Table 3, Figure 2). 2,24,25,38,39 Indacaterolglycopyrronium significantly improved transition dyspnea index (TDI) scores in SHINE and ILLUMINATE versus placebo, open-label tiotropium, and SFC. ${ }^{2,39}$ In BLAZE, indacaterol-glycopyrronium significantly improved selfadministered computerized total TDI score versus placebo (LSM treatment difference 1.37, $P<0.001$ ) and blinded tiotropium (LSM treatment difference: $0.49, P=0.021$ ). ${ }^{38}$ The proportion of patients achieving the MCID ( $\geq 1$-point) for TDI score was also significantly increased versus blinded

Table 3 Symptoms: margin of efficacy of fixed combinations versus comparators in published studies

\begin{tabular}{|c|c|c|c|c|c|}
\hline \multirow{2}{*}{$\begin{array}{l}\text { Reference } \\
\text { and study }\end{array}$} & \multirow[t]{2}{*}{ Duration } & \multirow[t]{2}{*}{ Treatment } & \multicolumn{3}{|c|}{ Treatment difference at end point } \\
\hline & & & $\begin{array}{l}\text { TDI total score, } \\
\text { LSM ( } 95 \% \mathrm{CI})\end{array}$ & $\begin{array}{l}\% \text { TDI } \\
\text { responders }{ }^{\mathrm{a}}(\mathrm{OR})\end{array}$ & Other \\
\hline \multicolumn{6}{|l|}{ IND-GLY } \\
\hline $\begin{array}{l}\text { Bateman et } \mathrm{al}^{2} \\
(\mathrm{SHINE})\end{array}$ & 26 weeks & $\begin{array}{l}\text { IND-GLY I I } 0 / 50 \mu \mathrm{g} \text { OD vs } \\
\text { Indacaterol I } 50 \mu \mathrm{g} \text { OD } \\
\text { Glycopyrronium } 50 \mu \mathrm{g} \text { OD } \\
\text { Tiotropium I } 8 \mu \mathrm{g} \text { OD OL } \\
\text { Placebo }\end{array}$ & $\begin{array}{l}0.25(\mathrm{NR}) \\
0.20(\mathrm{NR}) \\
0.5 \mathrm{I}^{\mathrm{b}}(\mathrm{NR}) \\
1.09^{\mathrm{b}}(0.6 \mathrm{I}-\mathrm{I} .57)\end{array}$ & $\begin{array}{l}3.5(\mathrm{NR}) \\
4.4(\mathrm{NR}) \\
8.9^{\mathrm{b}}(\mathrm{NR}) \\
10.6^{\mathrm{b}}(\mathrm{NR})\end{array}$ & $\begin{array}{l}\text { Diary data (values vs placebo): } \% \text { days with } \\
\text { no daytime symptoms, }+3.05^{b} ; \% \text { days able to } \\
\text { perform usual daily activities, }+1 \mathrm{I} .48^{\mathrm{b}-\mathrm{e}} ; \% \text { nights } \\
\text { without awakenings, }+10.0 \mathrm{I}^{\mathrm{b}, \mathrm{c}}\end{array}$ \\
\hline $\begin{array}{l}\text { Dahl et } \text { al }^{36} \\
(\text { ENLIGHTEN) }\end{array}$ & 52 weeks & $\begin{array}{l}\text { IND-GLY I I 0/50 } \mu \mathrm{g} \text { OD vs } \\
\text { Placebo }\end{array}$ & NR & NR & $\begin{array}{l}\text { Diary data: } \\
\text { Total daily symptom score, }-0.573^{\mathrm{b}} ; \% \text { days } \\
\text { with no daytime symptoms, }+5.3^{\mathrm{b}}, \% \text { days able } \\
\text { to perform usual daily activities, }+8.1^{\mathrm{b}} ; \% \text { nights } \\
\text { without awakenings, }+6.3\end{array}$ \\
\hline $\begin{array}{l}\text { Dahl et al }{ }^{37} \\
(\mathrm{BEACON})\end{array}$ & 4 weeks & $\begin{array}{l}\text { IND-GLY I I 0/50 } \mu \mathrm{g} \text { OD vs } \\
\text { indacaterol I } 50 \mu \mathrm{g} \text { OD + } \\
\text { glycopyrronium } 50 \mu \mathrm{g} \text { OD }\end{array}$ & NR & NR & $\begin{array}{l}\text { Diary data: } \\
\text { Total daily symptom score, } \\
0.07(-0.24,0.39)\end{array}$ \\
\hline $\begin{array}{l}\text { Mahler et } \mathrm{al}^{38} \\
\text { (BLAZE) }\end{array}$ & 6 weeks & $\begin{array}{l}\text { IND-GLY I I 0/50 } \mu \mathrm{g} \text { OD vs } \\
\text { tiotropium } 18 \mu \mathrm{g} \text { OD } \\
\text { Placebo }\end{array}$ & $\begin{array}{l}\text { SAC TDI: } \\
0.49^{\mathrm{b}}(0.07,0.91) \\
1.37^{\mathrm{b}}(0.95,1.79)\end{array}$ & $\begin{array}{l}\text { SAC TDI: } \\
\text { II. } 5^{\mathrm{b}}(2.78) \\
\mathrm{I} 7.8^{\mathrm{b}}(1.78)\end{array}$ & $\begin{array}{l}\text { Diary data (vs placebo and tiotropium): } \\
\text { Total daily symptom score, }-0.72^{\mathrm{b}} \text { and }-0.03 ; \\
\% \text { days with no daytime symptoms, }+3.5^{\mathrm{b}} \\
\text { and }+1.5 ; \% \text { nights with no awakenings, } 5.6^{\mathrm{b}} \text { and } \\
2.6 ; \text { days able to perform usual activities, } \\
8.8^{\mathrm{b}} \text { and }-0.4\end{array}$ \\
\hline $\begin{array}{l}\text { Vogelmeier } \\
\text { et } \text { al }^{39} \\
\text { (ILLUMINATE) }\end{array}$ & 26 weeks & $\begin{array}{l}\text { IND-GLY I I 0/50 } \mu \mathrm{g} \text { OD vs } \\
\text { SFC } 50 / 500 \mu \mathrm{g} \text { BID }\end{array}$ & $0.76^{\mathrm{b}}(0.26,1.26)$ & $10.7^{b}(1.56)$ & $\begin{array}{l}\text { Diary data: } \\
\text { Differences in scores for most symptoms NS } \\
\text { between treatment groups } \\
\% \text { days with no daytime symptoms, }+2.50^{\mathrm{b}}\end{array}$ \\
\hline $\begin{array}{l}\text { Beeh et a }{ }^{35} \\
(\text { BRIGHT) }\end{array}$ & 3 weeks & $\begin{array}{l}\text { IND-GLY I I 0/50 } \mu \mathrm{g} \text { OD vs } \\
\text { tiotropium } 18 \mu \mathrm{g} \text { OD } \\
\text { Placebo }\end{array}$ & $\begin{array}{l}N R \\
N R\end{array}$ & $\begin{array}{l}\text { NR } \\
\text { NR }\end{array}$ & $\begin{array}{l}\text { Diary data, mean daily symptom score vs } \\
\text { baseline: } \\
\text { IND-GLY }-0.64 \text {, tiotropium }-0.43 \text {, } \\
\text { placebo }-0.19\end{array}$ \\
\hline $\begin{array}{l}\text { Buhl et } \mathrm{al}^{25} \\
\text { (QUANTIFY) }\end{array}$ & 26 weeks & $\begin{array}{l}\text { IND-GLY I I 0/50 } \mu \mathrm{g} \text { OD vs } \\
\text { tiotropium } 18 \mu \mathrm{g} \text { OD + } \\
\text { formoterol } 12 \mu \mathrm{g} \text { BID }\end{array}$ & $0.38(-0.06,0.82)$ & $\begin{array}{l}7.2(\mathrm{I} .17 \text { risk } \\
\text { ratio })^{\mathrm{b}}\end{array}$ & $\begin{array}{l}\text { LSM treatment difference in SGRQ-C symptom } \\
\text { score IND-GLY vs tiotropium + formoterol } \\
(-1.31[95 \% \mathrm{Cl}-3.49,0.86])\end{array}$ \\
\hline $\begin{array}{l}\text { Zhong et a }{ }^{24} \\
\text { (LANTERN) }\end{array}$ & 26 weeks & $\begin{array}{l}\text { IND-GLY I I 0/50 } \mu \mathrm{g} \text { OD vs } \\
\text { SFC 50/500 } \mu \mathrm{g} \text { BID }\end{array}$ & $0.25(-0.09,0.59)$ & NR & $\begin{array}{l}\text { Improvements in TDI focal score at weeks I2 } \\
\text { and } 26 \text { similar between IND-GLY and SFC } \\
\text { Similar improvement in SGRQ total score } \\
\text { between IND-GLY and SFC at weeks } 12 \text { and } 26 \\
\text { Symptoms, rescue medication use and total } \\
\text { COPD assessment test scores at week } 26 \\
\text { comparable for IND-GLY and SFC }\end{array}$ \\
\hline UMEC-VI & & & & & \\
\hline Donohue et $\mathrm{al}^{41}$ & 24 weeks & $\begin{array}{l}\text { UMEC-VI } 62.5 / 25 \mu \mathrm{g} \text { OD vs } \\
\text { umeclidinium } 62.5 \mu \mathrm{g} \text { OD } \\
\text { Vilanterol } 25 \mu \mathrm{g} \text { OD } \\
\text { Placebo }\end{array}$ & $\begin{array}{l}0.3(-0.2,0.7) \\
0.4(-1.0,0.8) \\
1.2^{\mathrm{b}}(0.7,1.7)\end{array}$ & $\begin{array}{l}5.0(\mathrm{NR}) \\
7.0^{\mathrm{b}}(1.4) \\
17.0^{\mathrm{b}}(2.0)\end{array}$ & NR \\
\hline
\end{tabular}


Table 3 (Continued)

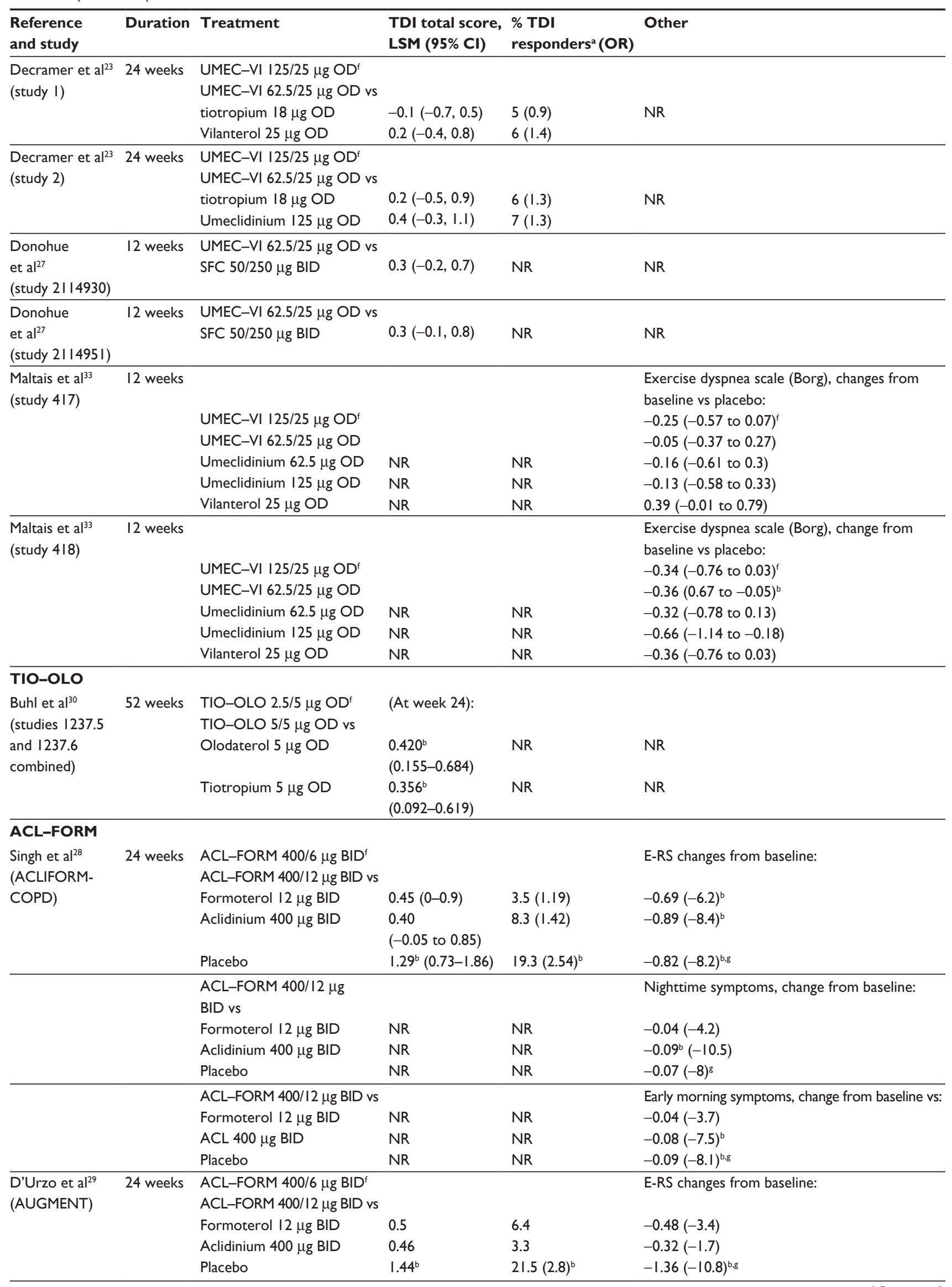


Table 3 (Continued)

\begin{tabular}{|c|c|c|c|c|c|}
\hline \multirow[t]{13}{*}{$\begin{array}{l}\text { Reference } \\
\text { and study }\end{array}$} & Duration & Treatment & $\begin{array}{l}\text { TDI total score, } \\
\text { LSM }(95 \% \mathrm{CI})\end{array}$ & $\begin{array}{l}\text { \% TDI } \\
\text { responders }{ }^{\mathrm{a}} \text { (OR) }\end{array}$ & Other \\
\hline & & ACL-FORM 400/6 $\mu g$ BID $^{f}$ & & & Night-time symptoms, change from baseline vs: \\
\hline & & ACL-FORM 400/I2 $\mu \mathrm{g}$ & & & \\
\hline & & BID vs & & & \\
\hline & & Formoterol $12 \mu \mathrm{g}$ BID & NR & NR & $-0.05(-2.4)$ \\
\hline & & Aclidinium $400 \mu \mathrm{g}$ BID & NR & NR & $-0.08(-5.3)$ \\
\hline & & Placebo & NR & NR & $-0.12(-9.3)^{\mathrm{b}, \mathrm{g}}$ \\
\hline & & ACL-FORM 400/6 $\mu \mathrm{g}$ BID $^{f}$ & & & Early-morning symptoms, \\
\hline & & ACL-FORM 400/I2 $\mu \mathrm{g}$ & & & change from baseline vs: \\
\hline & & BID vs & & & \\
\hline & & Formoterol $12 \mu \mathrm{g}$ BID & NR & NR & $-0.06(-4.6)$ \\
\hline & & Aclidinium $400 \mu \mathrm{g}$ BID & NR & NR & $-0.09(-7.4)^{\mathrm{b}}$ \\
\hline & & Placebo & NR & NR & $-0.13(-9.8)^{\mathrm{b}, \mathrm{g}}$ \\
\hline
\end{tabular}

Notes: Treatment once daily unless stated otherwise. ${ }^{a} \mathrm{TDI}$ responders had improvement $\geq \mathrm{I}$ unit in TDI score. ${ }^{\mathrm{b}}$ Significant treatment difference. Significant treatment difference versus cindacaterol, ${ }^{d}$ glycopyrronium or ${ }^{e}$ tiotropium (values NR). ${ }^{f}$ Dose not approved for use (ACL-FORM, dose not approved in EU). ${ }^{g} \mathrm{Values}$ in parentheses are differences expressed in percentage points (not percentage differences).

Abbreviations: ACL-FORM, aclidinium-formoterol; BID, bis in die (twice daily); Cl, confidence interval; E-RS, Evaluating Respiratory Symptoms; IND-GLY, indacaterolglycopyrronium; LSM, least-squares mean; NR, not reported; NS, not significant; OR, odds ratio; SAC, self-administered, computerized; SFC, salmeterol-fluticasone combination; SGRQ-C, St George's Respiratory Questionnaire - COPD; TIO-OLO, tiotropium-olodaterol; TDI, transition dyspnea index; UMEC-VI, umeclidiniumvilanterol.

tiotropium in BLAZE (OR 1.78, $P<0.05)$ and versus SFC in ILLUMINATE (OR 1.56, $P<0.05$; Figure 2). ${ }^{2,39}$ In QUANTIFY, a similar reduction in dyspnea was observed with indacaterol-glycopyrronium versus tiotropium + formoterol, and significantly more patients achieved clinically relevant improvements in TDI total score with indacaterol-glycopyrronium $(49.6 \%)$ versus tiotropium + formoterol $(42.4 \%, P=0.033) .{ }^{25}$

In LANTERN, a comparable improvement with indacaterol-glycopyrronium OD and SFC BID was demonstrated for TDI focal score and St George's Respiratory Questionnaire (SGRQ) total score from baseline after 26 weeks; the percentage of patients achieving the MCID for both end points was higher with indacaterol-glycopyrronium versus SFC. ${ }^{24}$ Compared with its component monotherapies, indacaterol-glycopyrronium was associated with numerical improvements in TDI score and percentage of TDI responders at week 26 in SHINE. ${ }^{2}$ At week 12, improvement in TDI score with indacaterol-glycopyrronium was significantly greater than with glycopyrronium (LSM treatment difference $0.41, P=0.03$ ).

Three indacaterol-glycopyrronium OD studies evaluated patient-diary data and reported significantly improved symptom scores versus indacaterol, glycopyrronium, tiotropium, or placebo (Table 3). 2,36,38 In the shorter BRIGHT trial, change in mean daily symptom score from baseline to week 3 was numerically greater for indacaterol-glycopyrronium versus tiotropium and placebo. ${ }^{35}$ In ILLUMINATE, differences in scores for most symptoms were comparable for indacaterolglycopyrronium and SFC BID. ${ }^{39}$
In three 24-week studies, umeclidinium-vilanterol $62.5 / 25 \mu \mathrm{g}$ OD significantly improved TDI focal and Shortness of Breath with Daily Activity (SOBDA) scores versus placebo, with numerical improvements versus monocomponents and tiotropium. ${ }^{23,41}$ The proportion of patients achieving the MCID for TDI score was significantly increased in patients receiving umeclidinium-vilanterol versus placebo (OR 2, $P<0.001)$ and vilanterol (OR 1.4, $P<0.05)^{41}$ in one study (Figure 2). ${ }^{23}$ LSM changes from baseline to week 24 in SOBDA scores were clinically significant ( $\geq 0.1$ unit) for umeclidinium-vilanterol, vilanterol $25 \mu \mathrm{g}$, umeclidinium 62.5 and $125 \mu \mathrm{g}$, and tiotropium $18 \mu \mathrm{g} .{ }^{23,41}$ SOBDA responder rates were reported for one trial, and were significantly higher for umeclidinium-vilanterol $(\mathrm{OR} 1.8, P<0.01)$ and its monocomponents (umeclidinium 52.5 $\mu \mathrm{g}$ OR 1.7, $P<0.01$; vilanterol $25 \mu \mathrm{g}$ OR 1.6, $P<0.05)$ versus placebo. In two 12 -week studies, there was no significant difference in TDI focal scores between umeclidinium-vilanterol 62.5/25 $\mu \mathrm{g}$ and salmeterol-fluticasone propionate $50 / 250 \mu \mathrm{g} .{ }^{27}$ Exerciseassociated dyspnea (Borg) was reduced with umeclidiniumvilanterol $62.5 / 25 \mu \mathrm{g}$ compared with placebo in one of two studies; active-placebo differences were not significant for the individual components. ${ }^{33}$ In combined results from two 1-year studies, tiotropium-olodaterol OD increased TDI total score versus monocomponents (week 24) by approximately 0.4 points with the higher dose and by a similar margin (0.3-0.4 points) with the lower dose. ${ }^{30}$

Symptoms were evaluated using a number of end points in the two 24-week aclidinium-formoterol BID studies. ${ }^{28,29}$ For TDI total score, aclidinium-formoterol 400/12 $\mu \mathrm{g}$ 

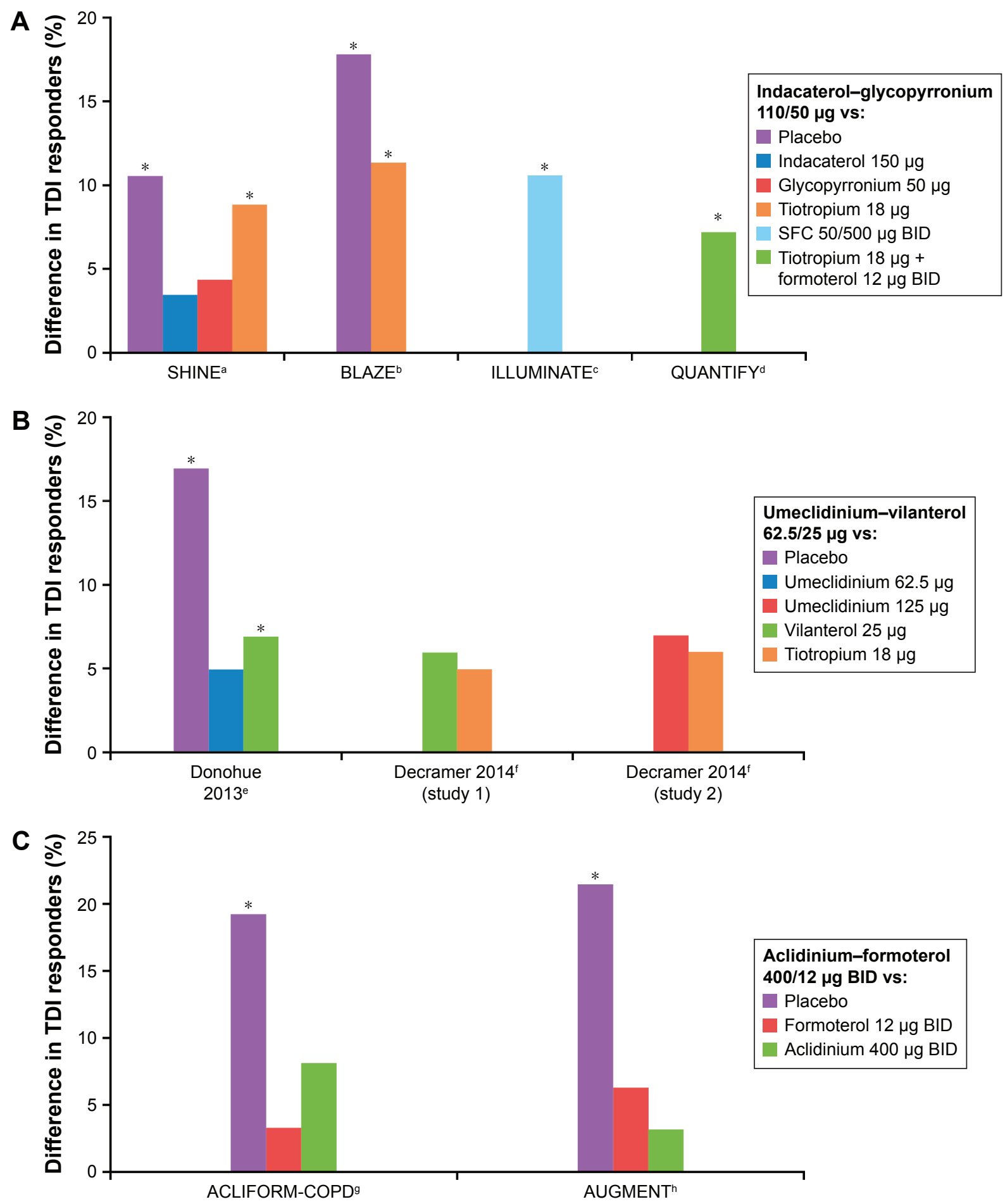

Figure 2 Differences between monotherapy and combination bronchodilators or placebo in TDI patient-response rates in published studies.

Notes: (A) Indacaterol-glycopyrronium; (B) umeclidinium-vilanterol 62.5/25 $\mu$; (C) aclidinium-formoterol 400/I2 $\mu \mathrm{g}$ BID. TDI response was defined as improvement of $\geq \mathrm{I}$ unit in TDI score. All treatments were once daily unless stated otherwise. *Significant treatment difference. ${ }^{\mathrm{a} B a t e m a n}$ et al; ${ }^{2}$ bself-administered computerized TDI; ${ }^{38}$ 'Vogelmeier et al; ${ }^{39}{ }^{\mathrm{d}}$ Buhl et al; ${ }^{25}$ eDonohue et al; ${ }^{41}$ fDecramer et al;;3 8 Singh et al;; ${ }^{28}$ 'D'Urzo et al. ${ }^{29}$

Abbreviations: BID, bis in die (twice daily); SFC, salmeterol-fluticasone propionate; TDI, transition dyspnea index.

BID achieved a significant, $>1$-point improvement versus placebo (and a higher proportion of TDI responders), but the differences versus the monotherapies were not significant. For Evaluating Respiratory Symptoms (E-RS) score, the combination was significantly better than placebo (both studies) and the monotherapies (one study). Aclidinium-formoterol 400/12 $\mu \mathrm{g}$ improved nighttime symptom scores versus placebo (one study) or aclidinium BID (one study); early morning symptom scores were improved versus placebo and aclidinium (both studies), assessed by questionnaires for both. ${ }^{28,29}$ 


\section{Rescue-medication use}

Rescue-medication usage provides a surrogate measure of symptom control, and was reported in most of the published indacaterol-glycopyrronium OD and umeclidiniumvilanterol OD Phase III trials (Table 4). Indacaterolglycopyrronium treatment consistently led to significantly less rescue-medication use per day than LABA or LAMA monotherapy or LABA-inhaled corticosteroids in each trial with active comparators..$^{2,26,35,38-40}$ In LANTERN, rescue-medication use was comparable between the indacaterol-glycopyrronium and SFC BID groups. ${ }^{24}$ Daily rescue-medication use was similar or numerically slightly lower with umeclidinium-vilanterol versus either umeclidinium or vilanterol monotherapy, significantly lower versus tiotropium in two of three trials, and significantly lower versus SFC in one of two trials. . $3,27,32,33,41$ Rescuemedication use remained at approximately two puffs/day with tiotropium-olodaterol OD over the course of 52 weeks; at the

Table 4 Rescue-medication use: margin of efficacy of fixed combinations versus comparators in published studies

\begin{tabular}{|c|c|c|c|}
\hline Reference and study & Duration & Treatment & $\begin{array}{l}\text { Rescue albuterol/salbutamol puffs/day } \\
\text { change from baseline, LSM }(95 \% \mathrm{Cl}) \\
\text { treatment difference at end point }\end{array}$ \\
\hline \multicolumn{4}{|l|}{ IND-GLY } \\
\hline \multirow[t]{5}{*}{ Bateman et $\mathrm{al}^{2}$ (SHINE) } & 26 weeks & IND-GLY I I0/50 $\mu \mathrm{g}$ OD vs & \\
\hline & & Indacaterol I $50 \mu \mathrm{g}$ OD & $-0.3 \mathrm{I}^{\mathrm{a}}(\mathrm{NR})$ \\
\hline & & Glycopyrronium $50 \mu \mathrm{g}$ OD & $-0.66^{\mathrm{a}}(\mathrm{NR})$ \\
\hline & & Tiotropium I $8 \mu \mathrm{g}$ OD OL & $-0.55^{\mathrm{a}}(\mathrm{NR})$ \\
\hline & & Placebo & $-0.96^{\mathrm{a}}(-1.29$ to -0.62$)$ \\
\hline \multirow[t]{2}{*}{ Dahl et a ${ }^{36}$ (ENLIGHTEN) } & 52 weeks & IND-GLY I I 0/50 $\mu \mathrm{g}$ OD vs & \\
\hline & & Placebo & $-0.73^{\mathrm{a}}(\mathrm{NR})$ \\
\hline \multirow[t]{2}{*}{ Dahl et $\mathrm{al}^{37}$ (BEACON) } & 4 weeks & IND-GLY I I 0/50 $\mu \mathrm{g}$ OD vs & \\
\hline & & $\begin{array}{l}\text { Indacaterol I } 50 \mu \mathrm{g} \text { OD + } \\
\text { glycopyrronium } 50 \mu \mathrm{g} \text { OD }\end{array}$ & $-0.04(-0.35$ to 0.28$)$ \\
\hline \multirow[t]{3}{*}{ Mahler et al ${ }^{38}$ (BLAZE) } & 6 weeks & IND-GLY I I 0/50 $\mu \mathrm{g}$ OD vs & \\
\hline & & Placebo & $-1.43^{\mathrm{a}}(-1.72$ to -1.13$)$ \\
\hline & & Tiotropium I $8 \mu \mathrm{g}$ OD & $-0.45^{\mathrm{a}}(-0.74$ to -0.16$)$ \\
\hline \multirow[t]{2}{*}{ Vogelmeier et al ${ }^{39}$ (ILLUMINATE) } & 26 weeks & IND-GLY I I 0/50 $\mu \mathrm{g}$ OD vs & \\
\hline & & SFC 50/500 $\mu \mathrm{g}$ BID & $-0.39^{\mathrm{a}}(-0.7 \mathrm{I}$ to -0.06$)$ \\
\hline \multirow[t]{3}{*}{ Wedzicha et al ${ }^{40}$ (SPARK) } & 64 weeks & IND-GLY I I 0/50 $\mu \mathrm{g}$ OD vs & \\
\hline & & Glycopyrronium $50 \mu \mathrm{g}$ OD & $-0.8 \mathrm{I}^{\mathrm{a}}(\mathrm{NR})$ \\
\hline & & Tiotropium $18 \mu \mathrm{g}$ OD OL & $-0.76^{\mathrm{a}}(\mathrm{NR})$ \\
\hline \multirow[t]{3}{*}{ Beeh et $\mathrm{a}^{35}$ (BRIGHT) } & 3 weeks & IND-GLY I I0/50 $\mu \mathrm{g}$ OD vs & \\
\hline & & Tiotropium $18 \mu \mathrm{g}^{\mathrm{a}}$ OD & $-1.08^{\mathrm{a}}(\mathrm{NR})$ \\
\hline & & Placebo & $-1.23^{\mathrm{a}}(\mathrm{NR})$ \\
\hline \multirow[t]{2}{*}{ Zhong et al ${ }^{24}$ (LANTERN) } & 26 weeks & IND-GLY I I0/50 $\mu \mathrm{g}$ OD vs & \\
\hline & & SFC $50 / 500 \mu \mathrm{g}$ BID & $-0.03(-0.26$ to 0.21$)$ \\
\hline \multirow[t]{2}{*}{ Wedzicha et al ${ }^{26}$ (FLAME) } & 52 weeks & IND-GLY I I 0/50 $\mu \mathrm{g}$ OD vs & \\
\hline & & SFC $50 / 500 \mu \mathrm{g}$ BID & $-0.25^{\mathrm{a}}(-0.38$ to -0.12$)$ \\
\hline \multicolumn{4}{|l|}{ UMEC-VI } \\
\hline \multirow[t]{4}{*}{ Donohue et $\mathrm{a}^{41}$} & 24 weeks & UMEC-VI $62.5 / 25 \mu \mathrm{g}$ OD vs & \\
\hline & & Umeclidinium $62.5 \mu \mathrm{g}$ OD & $-0.6^{\mathrm{a}}(-1.0$ to -0.1$)$ \\
\hline & & Vilanterol $25 \mu \mathrm{g}$ OD & $0.1(-0.3$ to 0.5$)$ \\
\hline & & Placebo & $-0.8^{\mathrm{a}}(-1.3$ to -0.3$)$ \\
\hline \multirow[t]{4}{*}{ Decramer et al ${ }^{23}$ (study I) } & 24 weeks & UMEC-VI I $25 / 25 \mu \mathrm{g} \mathrm{OD}^{\mathrm{b}}$ & \\
\hline & & UMEC-VI $62.5 / 25 \mu \mathrm{g}$ OD vs & \\
\hline & & Tiotropium I8 $\mu \mathrm{g}$ OD & $-0.7^{\mathrm{a}}(-1.2$ to -0.1$)$ \\
\hline & & Vilanterol $25 \mu \mathrm{g}$ OD & $-0.3(-0.8$ to 0.3$)$ \\
\hline \multirow[t]{4}{*}{ Decramer et al ${ }^{23}$ (study 2 ) } & 24 weeks & UMEC-VI I 25/25 $\mu \mathrm{g}$ OD $^{\mathrm{b}}$ & \\
\hline & & UMEC-VI $62.5 / 25 \mu \mathrm{g}$ OD vs & \\
\hline & & Tiotropium I8 $\mu \mathrm{g}$ OD & $-0.6(-1.2$ to 0$)$ \\
\hline & & Umeclidinium I $25 \mu \mathrm{g}$ OD & $-0.6(-1.2$ to 0$)$ \\
\hline \multirow[t]{2}{*}{ Maleki-Yazdi et al ${ }^{32}$} & 24 weeks & UMEC-VI $62.5 / 25 \mu \mathrm{g}$ OD vs & \\
\hline & & Tiotropium I8 $\mu \mathrm{g}$ OD & $-0.5^{\mathrm{a}}(-0.7$ to -0.2$)$ \\
\hline
\end{tabular}

(Continued) 
Table 4 (Continued)

\begin{tabular}{|c|c|c|c|}
\hline Reference and study & Duration & Treatment & $\begin{array}{l}\text { Rescue albuterol/salbutamol puffs/day } \\
\text { change from baseline, LSM }(95 \% \mathrm{Cl}) \\
\text { treatment difference at end point }\end{array}$ \\
\hline \multirow[t]{5}{*}{ Maltais et al $\left.\right|^{33}$ (study 4I7) } & 12 weeks & UMEC-VI I25/25 $\mu \mathrm{g} \mathrm{OD}^{\mathrm{b}}$ & Differences from placebo: \\
\hline & & UMEC-VI 62.5/25 $\mu \mathrm{g}$ OD & $-0.6^{\mathrm{a}}(-0.8$ to -0.3$)$ \\
\hline & & Umeclidinium $62.5 \mu \mathrm{g}$ OD & $-0.2(-0.6$ to 0.1$)$ \\
\hline & & Umeclidinium I $25 \mu \mathrm{g}$ OD & $-0.6^{a}(-1$ to -0.2$)$ \\
\hline & & Vilanterol $25 \mu \mathrm{g}$ OD & $-0.4^{a}(-0.7$ to 0$)$ \\
\hline \multirow[t]{5}{*}{ Maltais et al ${ }^{33}$ (study 4I8) } & 12 weeks & UMEC-VI I25/25 $\mu \mathrm{g} \mathrm{OD}^{\mathrm{b}}$ & Differences from placebo: \\
\hline & & UMEC-VI 62.5/25 $\mu \mathrm{g}$ OD & $-1.2^{\mathrm{a}}(-1.5$ to -0.8$)$ \\
\hline & & Umeclidinium $62.5 \mu \mathrm{g}$ OD & $-0.7^{\mathrm{a}}(-1.3$ to -0.2$)$ \\
\hline & & Umeclidinium I $25 \mu \mathrm{g}$ OD & $-1.0^{\mathrm{a}}(-1.5$ to -0.4$)$ \\
\hline & & Vilanterol $25 \mu \mathrm{g}$ OD & $-0.8^{\mathrm{a}}(-1.2$ to -0.3$)$ \\
\hline \multirow[t]{2}{*}{ Donohue et al ${ }^{27}$ (study 2 II4930) } & 12 weeks & UMEC-VI $62.5 / 25 \mu \mathrm{g}$ OD vs & \\
\hline & & SFC $50 / 250 \mu \mathrm{g}$ BID & $0(-0.3$ to 0.2$)$ \\
\hline \multirow[t]{2}{*}{ Donohue et al ${ }^{27}$ (study 2 II495I) } & 12 weeks & UMEC-VI $62.5 / 25 \mu \mathrm{g}$ OD vs & \\
\hline & & SFC 50/250 $\mu \mathrm{g}$ BID & $-0.3^{\mathrm{a}}(-0.6$ to -0.1$)$ \\
\hline \multicolumn{4}{|l|}{ TIO-OLO } \\
\hline Buhl et al ${ }^{10}$ (studies 1237.5 and & 52 weeks & TIO-OLO 2.5/5 $\mu \mathrm{g} \mathrm{OD}^{\mathrm{b}}$ & \\
\hline \multirow[t]{3}{*}{ I 237.6 combined) } & & TIO-OLO $5 / 5 \mu \mathrm{g}$ OD vs & \\
\hline & & Olodaterol $5 \mu \mathrm{g}$ OD & $\sim-0.4^{c}$ \\
\hline & & Tiotropium $5 \mu \mathrm{g}$ OD & $\sim-0.8^{c}$ \\
\hline \multicolumn{4}{|l|}{ ACL-FORM } \\
\hline \multirow[t]{6}{*}{ Singh et a ${ }^{28}$ (ACLIFORM-COPD) } & 24 weeks & ACL-FORM 400/6 $\mu \mathrm{g}$ BID $^{\mathrm{b}}$ & \\
\hline & & ACL-FORM 400/12 $\mu \mathrm{g}$ & \\
\hline & & BID vs & \\
\hline & & Formoterol I $2 \mu \mathrm{g}$ BID & NS \\
\hline & & Aclidinium $400 \mu \mathrm{g}$ BID & Value $\mathrm{NR}^{\mathrm{a}}$ \\
\hline & & Placebo & $-0.66^{\mathrm{a}}$ \\
\hline \multirow[t]{6}{*}{ D’Urzo et a ${ }^{29}$ (AUGMENT) } & 24 weeks & ACL-FORM 400/6 $\mu \mathrm{g}$ BID $^{b}$ & \\
\hline & & ACL-FORM 400/I2 $\mu \mathrm{g}$ & \\
\hline & & BID vs & \\
\hline & & Formoterol I $2 \mu \mathrm{g}$ BID & 0.21 \\
\hline & & Aclidinium $400 \mu \mathrm{g}$ BID & $0.43^{\mathrm{a}}$ \\
\hline & & Placebo & Value $N^{a}$ \\
\hline
\end{tabular}

Notes: Treatment once daily unless stated otherwise. a Significant treatment difference; bdose not approved for use; 'estimated from figure. Statistical analysis not reported. Abbreviations: ACL-FORM, aclidinium-formoterol; BID, bis in die (twice daily); Cl, confidence interval; IND-GLY, indacaterol-glycopyrronium; LSM, least-squares mean; NR, not reported; NS, not significant; OL, open-label; SFC, salmeterol-fluticasone combination; TIO-OLO, tiotropium-olodaterol; UMEC-VI, umeclidinium-vilanterol.

end of the studies, this was $0.3-0.4$ puffs/day less than with olodaterol and $0.7-0.8$ puffs/day less than with tiotropium. ${ }^{30}$ In the two 24-week studies with aclidinium-formoterol $400 / 12 \mu \mathrm{g}$ BID, rescue-medication use was significantly lower compared with placebo and aclidinium BID, but not compared with formoterol. ${ }^{28,29}$

\section{Exacerbations}

The effects of FDC therapy on exacerbation rates and time to first exacerbation are summarized in Table 5.

The effect of indacaterol-glycopyrronium OD on exacerbation rate was examined as the primary end point in both SPARK and FLAME, and exacerbation rates have also been reported from ILLUMINATE, LANTERN, and QUANTIFY. ${ }^{24-26,39,40,52}$ In SPARK, indacaterol-glycopyrronium significantly reduced rates of moderate-to-severe (primary end point, rate ratio $0.88 ; P=0.038$ ) and all exacerbations (LSM treatment difference $0.85, P<0.01$ ) versus glycopyrronium. ${ }^{40}$ Compared with open-label tiotropium, rates of moderate-to-severe exacerbations were $10 \%$ lower with indacaterol-glycopyrronium $(P=0.096)$, and rates of all exacerbations were $14 \%$ lower $(P<0.01)$. In comparison with SFC BID in a post hoc analysis of data from ILLUMINATE, rates of moderate-to-severe exacerbations (rate ratio 0.8 , not significant $[\mathrm{NS}]$ ) and all exacerbations (rate ratio 0.69 , NS) were numerically lower with indacaterol-glycopyrronium. ${ }^{52}$ In LANTERN, indacaterol-glycopyrronium significantly reduced the rate of moderate or severe exacerbations by $31 \%(P=0.048)$ over SFC. ${ }^{24}$ Furthermore, in the recent FLAME study, indacaterol-glycopyrronium significantly 


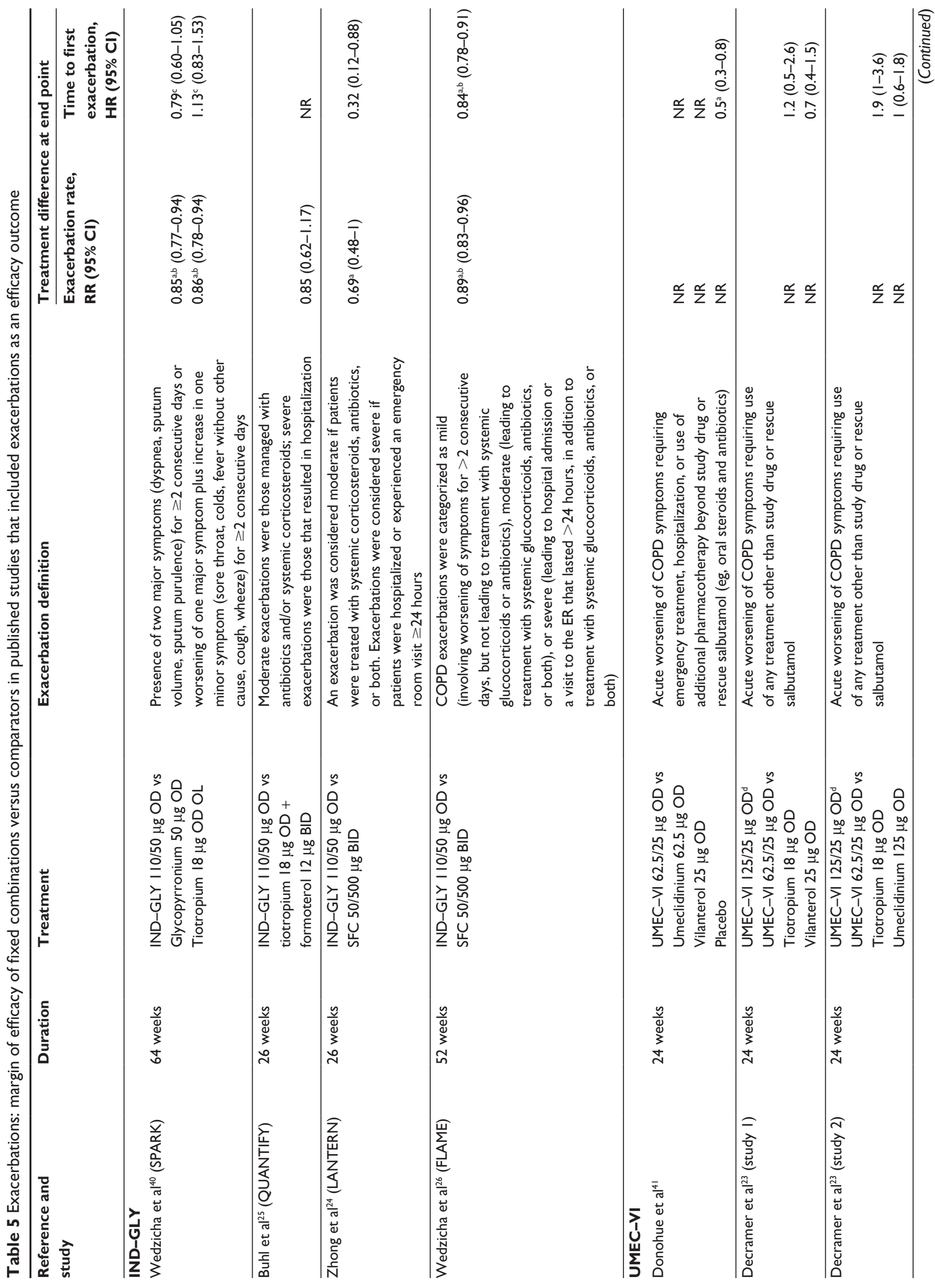




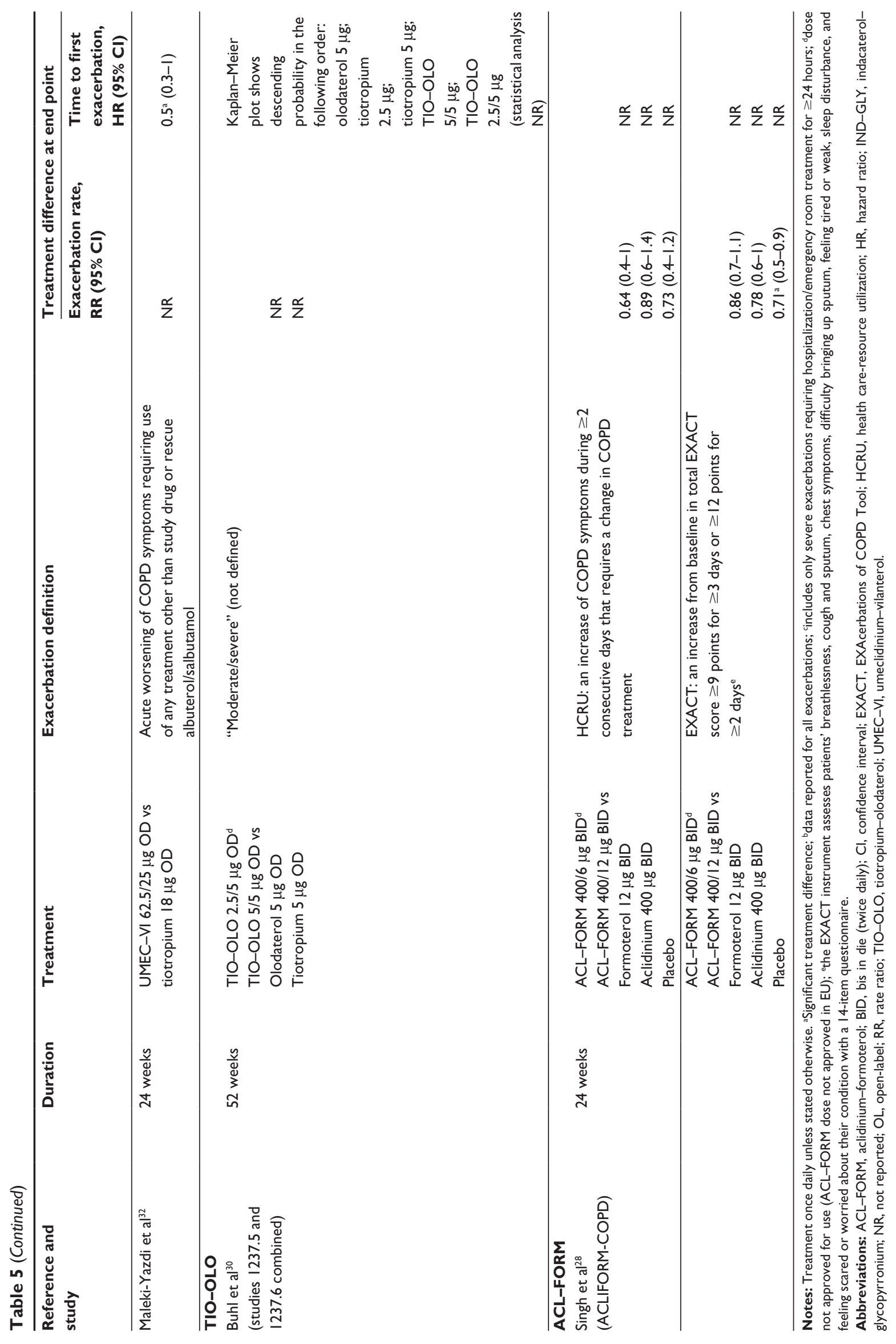


reduced the rates of all exacerbations (primary end point) by $11 \%(P=0.003)$ and of moderate-to-severe exacerbations by $17 \%(P<0.001)$ compared with SFC; findings were consistently in favor of indacaterol-glycopyrronium when patients were analyzed according to their baseline disease characteristics, including baseline eosinophil count $(<2 \%$ or $\geq 2 \%$ ). ${ }^{26}$ This study also found that compared with SFC, indacaterol-glycopyrronium was associated with longer times to first exacerbation, representing reduced risks of $16 \%$ for all exacerbations $(P<0.001), 22 \%$ for moderate-to-severe exacerbations $(P<0.001)$, and $19 \%$ for severe exacerbations $(P=0.046)$. Finally, QUANTIFY showed a comparable percentage of patients experiencing at least one moderate or severe exacerbation and a comparable time to first moderate or severe exacerbation between the two treatment groups (indacaterol-glycopyrronium vs tiotropium + formoterol). ${ }^{25}$

Currently, there are no studies evaluating exacerbation risk as a primary end point in patients receiving umeclidiniumvilanterol OD. The data available from analysis of secondary end points indicate that umeclidinium-vilanterol significantly increased time to first exacerbation versus placebo
(HR 0.5, $P<0.001$ ), ${ }^{41}$ but not compared with vilanterol 25 $\mu \mathrm{g}$ (HR 0.7, NS) or umeclidinium $125 \mu \mathrm{g}$ (HR 1, NS). ${ }^{23}$

Time to first exacerbation was comparable for combination therapy versus tiotropium alone in two trials $\mathrm{s}^{23}$ and significantly greater in a third study (HR $0.5, P=0.044) .{ }^{32}$ In the combined results of the two 52-week studies with tiotropiumolodaterol OD, there was only a "trend" for improvement in exacerbations with both doses of the combination versus the monotherapy components. ${ }^{30}$ Over the 24 weeks of the ACLIFORM study, using the health care resource-utilization definition of exacerbations, aclidinium-formoterol BID 400/12 $\mu \mathrm{g}$ was not significantly different from placebo or its separate components; with the EXACT (EXAcerbations of COPD Tool) definition, a significant difference was demonstrated versus placebo, but not compared with the components. ${ }^{28}$

Exacerbations were not reported as an efficacy outcome in the AUGMENT study. ${ }^{29}$

\section{Health status}

Indacaterol-glycopyrronium OD significantly improved health status, assessed using the SGRQ (Table 6). In SPARK,

Table 6 Health status: margin of efficacy of fixed combinations versus comparators in published studies

\begin{tabular}{|c|c|c|c|c|}
\hline \multirow{2}{*}{$\begin{array}{l}\text { Reference } \\
\text { and study }\end{array}$} & \multirow[t]{2}{*}{ Duration } & \multirow[t]{2}{*}{ Treatment } & \multicolumn{2}{|l|}{ Treatment difference at end point } \\
\hline & & & SGRQ total score, LSM (95\% Cl) & \% SGRQ responders (OR) \\
\hline \multicolumn{5}{|l|}{ IND-GLY } \\
\hline Bateman et $\mathrm{al}^{2}$ & 26 weeks & IND-GLY I I0/50 $\mu \mathrm{g}$ OD vs & & \\
\hline \multirow[t]{4}{*}{ (SHINE) } & & Indacaterol I50 $\mu \mathrm{g}$ OD & $-1.09(\mathrm{NR})$ & $0.7(\mathrm{NR})$ \\
\hline & & Glycopyrronium $50 \mu \mathrm{g}$ OD & $-1.18(N R)$ & $3.2(\mathrm{NR})$ \\
\hline & & Tiotropium $18 \mu \mathrm{g}$ OD OL & $-2.13^{\mathrm{a}}(\mathrm{NR})$ & $7.3^{\mathrm{a}}(\mathrm{NR})$ \\
\hline & & Placebo & $-3.0 \mathrm{I}^{\mathrm{a}}(-5.05$ to -0.97$)$ & $7 . I(N R)$ \\
\hline Vogelmeier et $\mathrm{a}^{39}$ & 26 weeks & IND-GLY I I 0/50 $\mu \mathrm{g}$ OD vs & & \\
\hline (ILLUMINATE) & & SFC $50 / 500 \mu g$ BID & $-1.24(-3.33$ to 0.85$)$ & $6.4(1.32)$ \\
\hline Wedzicha et al ${ }^{40}$ & 64 weeks & IND-GLY I I0/50 $\mu \mathrm{g}$ OD vs & & \\
\hline \multirow[t]{2}{*}{ (SPARK) } & & Glycopyrronium $50 \mu \mathrm{g}$ OD & -1.9 to $-2.8^{\mathrm{b}}(\mathrm{NR})$; all $P<0.0 \mathrm{I}$ & NR (I.28) \\
\hline & & Tiotropium $18 \mu \mathrm{g}$ OD OL & -1.7 to $-3 . I^{b}(N R) ;$ all $P<0.05$ & $N R(1.29)$ \\
\hline Buhl et $\mathrm{al}^{25}$ & 26 weeks & IND-GLY I I0/50 $\mu \mathrm{g}$ OD vs & & \\
\hline (QUANTIFY) & & $\begin{array}{l}\text { tiotropium I } 8 \mu \mathrm{g} \mathrm{OD} \mathrm{+} \mathrm{formoterol} \\
12 \mu \mathrm{g} \mathrm{BID}\end{array}$ & $-0.69(-2.31$ to 0.92$)$ & 4.5 (risk ratio I.I I) \\
\hline Zhong et $\mathrm{a}^{24}$ & 26 weeks & IND-GLY I I 0/50 $\mu \mathrm{g}$ OD vs & & \\
\hline (LANTERN) & & SFC $50 / 500 \mu \mathrm{g}$ BID & $-0.69(-2.38$ to $I)$ & NR \\
\hline Wedzicha et $\mathrm{a}^{26}$ & 52 weeks & IND-GLY I I 0/50 $\mu \mathrm{g}$ OD vs & & \\
\hline (FLAME) & & SFC $50 / 500 \mu \mathrm{g}$ BID & $-1.8^{\mathrm{a}}(\mathrm{NR})$ & I. $3^{\mathrm{a}}(\mathrm{NR})$ \\
\hline \multicolumn{5}{|l|}{ UMEC-VI } \\
\hline \multirow[t]{4}{*}{ Donohue et $\mathrm{al}^{41}$} & 24 weeks & UMEC-VI $62.5 / 25 \mu \mathrm{g}$ OD vs & Change from baseline: & \\
\hline & & Umeclidinium $62.5 \mu \mathrm{g}$ OD & $-0.82^{\mathrm{c}}(-2.90$ to $\mathrm{I} .27)$ & 5 (NR) \\
\hline & & Vilanterol $25 \mu \mathrm{g}$ OD & $-0.32^{\mathrm{c}}(-2.4 \mathrm{I}$ to $\mathrm{I} .78)$ & I (NR) \\
\hline & & Placebo & $-\left.5.5\right|^{\mathrm{a}, \mathrm{c}}(-7.88$ to -3.13$)$ & $15(2)^{\mathrm{a}}$ \\
\hline Decramer et al ${ }^{23}$ & 24 weeks & UMEC-VI I $25 / 25 \mu \mathrm{g} \mathrm{OD}^{\mathrm{d}}$ & Change from baseline: & \\
\hline \multirow[t]{3}{*}{ (study I) } & & UMEC-VI $62.5 / 25 \mu \mathrm{g}$ OD vs & & \\
\hline & & Tiotropium $18 \mu \mathrm{g}$ OD & $0.75^{c}(N R)$ & $3(0.9)$ \\
\hline & & Vilanterol $25 \mu \mathrm{g}$ OD & $\mathrm{I} .42^{\mathrm{c}}(\mathrm{NR})$ & $3(0.8)$ \\
\hline
\end{tabular}


Table 6 (Continued)

\begin{tabular}{|c|c|c|c|c|}
\hline \multirow{2}{*}{$\begin{array}{l}\text { Reference } \\
\text { and study }\end{array}$} & \multirow[t]{2}{*}{ Duration } & \multirow[t]{2}{*}{ Treatment } & \multicolumn{2}{|l|}{ Treatment difference at end point } \\
\hline & & & SGRQ total score, LSM $(95 \% \mathrm{Cl})$ & \% SGRQ responders (OR) \\
\hline \multirow{4}{*}{$\begin{array}{l}\text { Decramer et a }{ }^{23} \\
\text { (study 2) }\end{array}$} & 24 weeks & UMEC-VI I $25 / 25 \mu g$ OD $^{d}$ & Change from baseline: & \\
\hline & & UMEC-VI $62.5 / 25 \mu \mathrm{g}$ OD vs & & \\
\hline & & Tiotropium I8 $\mu \mathrm{g}$ OD & $-0.17^{c}(\mathrm{NR})$ & $\mathrm{I}(\mathrm{I})$ \\
\hline & & Umeclidinium I $25 \mu \mathrm{g}$ OD & $-1.55^{c}(\mathrm{NR})$ & $6(1.3)$ \\
\hline \multirow[t]{2}{*}{$\overline{\text { Maleki-Yazdi et al }{ }^{32}}$} & 24 weeks & UMEC-VI $62.5 / 25 \mu \mathrm{g} v \mathrm{~s}$ & Change from baseline: & \\
\hline & & tiotropium $18 \mu \mathrm{g}$ & $-2.1^{\mathrm{a}}(-3.61$ to -0.59$)$ & $7^{\mathrm{a}}(1.4)$ \\
\hline Donohue et $\mathrm{al}^{27}$ & 12 weeks & UMEC-VI $62.5 / 25 \mu \mathrm{g}$ OD vs & & \\
\hline (study 2114930 ) & & SFC $50 / 250 \mu \mathrm{g}$ BID & $0.47(-1.36$ to 2.29$)$ & NR \\
\hline Donohue et $\mathrm{al}^{27}$ & 12 weeks & UMEC-VI $62.5 / 25 \mu \mathrm{g}$ OD vs & & \\
\hline (study $21 \mid 4951$ ) & & SFC $50 / 250 \mu \mathrm{g}$ BID & $-1.55(-3.63$ to 0.53$)$ & NR \\
\hline \multicolumn{5}{|l|}{ TIO-OLO } \\
\hline Buhl et a ${ }^{30}$ (studies & 52 weeks & TIO-OLO 2.5/5 $\mu \mathrm{g} \mathrm{OD}^{\mathrm{d}}$ & At 24 weeks: & At 24 weeks: ${ }^{f}$ \\
\hline 1237.5 and 1237.6 & & TIO-OLO $5 / 5 \mu \mathrm{g}$ OD vs & & \\
\hline \multirow[t]{2}{*}{ combined) } & & Olodaterol $5 \mu \mathrm{g}$ OD & $-1.693^{\mathrm{a}, \mathrm{e}}$ & $12.7^{\mathrm{a}}$ \\
\hline & & Tiotropium $5 \mu \mathrm{g}$ OD & $-1.233^{\mathrm{a}}$ & $8.8^{\mathrm{a}}$ \\
\hline \multicolumn{5}{|l|}{ ACL-FORM } \\
\hline Singh et $\mathrm{a}^{28}$ & 24 weeks & ACL-FORM 400/6 $\mu \mathrm{g} \mathrm{BID}^{d}$ & Change from baseline: & \\
\hline (ACLIFORM- & & ACL-FORM $400 / 12 \mu \mathrm{g}$ BID vs & & \\
\hline \multirow[t]{3}{*}{ COPD) } & & Formoterol $12 \mu \mathrm{g}$ BID & $-1.59(-3.52$ to 0.35$)$ & NR \\
\hline & & Aclidinium $400 \mu \mathrm{g}$ BID & $-1.36(-3.3$ to 0.58$)$ & NR \\
\hline & & Placebo & $-0.65(-3.08$ to 1.78$)$ & NR \\
\hline$\overline{\text { D'Urzo et } \mathrm{al}^{29}}$ & 24 weeks & ACL-FORM 400/6 $\mu \mathrm{g} \mathrm{BID}^{\mathrm{d}}$ & Change from baseline: & \\
\hline \multirow[t]{4}{*}{ (AUGMENT) } & & ACL-FORM $400 / 12 \mu \mathrm{g}$ BID vs & & \\
\hline & & Formoterol $12 \mu \mathrm{g}$ BID & -1.87 & 5.8 \\
\hline & & Aclidinium $400 \mu \mathrm{g}$ BID & -0.13 & 3.7 \\
\hline & & Placebo & $-4.36^{\mathrm{a}}$ & $19.5(2.3)^{\mathrm{a}}$ \\
\hline
\end{tabular}

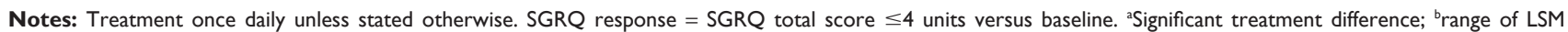
differences in scores for weeks I2, 24, 38, 52, and 64 (95\% CI not reported); 'differences in LSM change from baseline to week 24; dose not approved for use (ACL-FORM dose not approved in $\mathrm{EU})$; ${ }^{\mathrm{e}} 95 \% \mathrm{Cl}$ not reported; fOR not reported.

Abbreviations: ACL-FORM, aclidinium-formoterol; BID, bis in die (twice daily); Cl, confidence interval; IND-GLY, indacaterol-glycopyrronium; LSM, least-squares mean; NR, not reported; OL, open-label; OR, odds ratio; SFC, salmeterol-fluticasone combination; SGRQ, St George's Respiratory Questionnaire; TIO-OLO, tiotropiumolodaterol; UMEC-VI, umeclidinium-vilanterol.

indacaterol-glycopyrronium improved SGRQ total score versus glycopyrronium (all $P<0.01$ ) and open-label tiotropium (all $P<0.05 ; 12-64$ weeks)..$^{40}$ In SHINE, improvement in SGRQ with indacaterol-glycopyrronium was superior to open-label tiotropium $(P=0.009)$ and placebo $(P=0.002)$ and comparable to component monotherapies. ${ }^{2}$ In a 26 -week study, indacaterol-glycopyrronium and SFC BID provided similar improvements in health status. ${ }^{39}$ However, in FLAME, significant improvements over time in SGRQ total score were observed for indacaterol-glycopyrronium compared with SFC, with treatment differences that ranged from -1.2 points to -1.8 points over the time points measured between weeks 12 and 52 (all $P<0.01$ ). ${ }^{26}$ The SGRQ responder rate for the MCID (reduction of $\leq 4$ units from baseline) ${ }^{53}$ was also significantly greater with indacaterol-glycopyrronium versus SFC in FLAME (OR 1.3, $P<0.001)^{26}$ and versus glycopyrronium (OR 1.62, $P=0.00013$ ) and open-label tiotropium (OR 1.48, $P=0.0017$ ) at all time points except week 64 in SPARK. ${ }^{40}$ In QUANTIFY, indacaterol-glycopyrronium was noninferior to tiotropium + formoterol for improvement in SGRQ score; the percentage of patients achieving a MCID was significantly in favor of indacaterol-glycopyrronium $(50.1 \%$ vs $42.5 \%, P=0.038$ ) in the per-protocol set. ${ }^{25}$ Similarly, in LANTERN comparable improvements with indacaterolglycopyrronium versus SFC were observed for all SGRQ analyses (weeks 12 and 26). ${ }^{24}$

Significant improvements in SGRQ total score mean change from baseline $(P \leq 0.001)$ and percentages of SGRQ responders (OR 2, $P \leq 0.001$ ) were reported for umeclidinium-vilanterol $62.5 / 25 \mu \mathrm{g}$ OD versus placebo in three 24-week studies. ${ }^{41}$ Across three of four trials, health-status improvement was not significantly different for umeclidinium-vilanterol versus monotherapy with tiotropium, vilanterol, or umeclidinium (SGRQ total scores or percentage of SGRQ responders). ${ }^{23,41}$ The fourth trial reported significant improvement in SGRQ total score from baseline $(P<0.006)$ and percentage of SGRQ responders (OR 1.4, $P=0.022$ ) for umeclidinium-vilanterol versus 
tiotropium. ${ }^{32}$ Improvements in SGRQ from baseline were not significantly different between umeclidinium-vilanterol $62.5 / 25 \mu \mathrm{g}$ and salmeterol-fluticasone propionate $50 / 250 \mu \mathrm{g}$ in two 12-week studies. ${ }^{27}$

In combined results from two 1-year studies, tiotropiumolodaterol $5 / 5 \mu \mathrm{g}$ OD significantly improved SGRQ total score at week 24 by 1.2 and 1.7 units versus its respective components. Proportions of SGRQ responders were significantly increased for all the combination-versus-component comparisons, apart from tiotropium-olodaterol 2.5/5 $\mu \mathrm{g}$ versus tiotropium $2.5 \mu \mathrm{g}$. In the 24 -week ACLIFORM and AUGMENT studies, aclidinium-formoterol BID improved SGRQ total score and percentage of responders significantly compared with placebo in one study, but did not achieve significant differences against its components in either study. ${ }^{28,29}$

\section{Safety}

To date, the most extensive safety data available for FDC bronchodilators comes from indacaterol-glycopyrronium OD trials. Overall, indacaterol-glycopyrronium was well tolerated across the studies, and had a similar safety profile to placebo in individual trials and analyses of pooled data. ${ }^{2,36,39,40,54-56}$ The incidence of adverse events (AEs) and serious AEs (SAEs) reported with indacaterol-glycopyrronium treatment was comparable to that of placebo, indacaterol, glycopyrronium, tiotropium ( \pm formoterol) or SFC BID. ${ }^{2,24-26,36,39,40}$ Interestingly, the FLAME trial reported a significant reduction in the incidence of pneumonia with indacaterol-glycopyrronium compared with SFC (3.2\% vs $4.8 \%$, respectively; $P=0.02) .{ }^{26}$ In an analysis of pooled data from 11,404 patients, the HR for indacaterol-glycopyrronium versus placebo showed no significant increase in the overall risk for death (HR [95\% CI] 0.93 [0.34-2.54]), cardiocerebrovascular events (0.6 [0.29-1.24]), major adverse cardiovascular events (MACEs; 1.04 [0.45-2.42]), pneumonia (1.1 [0.54-2.25]), COPD exacerbations (0.6 [0.4-0.91]), or atrial flutter/fibrillation $(1.03[0.49-2.18]) .{ }^{54}$

Over 24 weeks, umeclidinium-vilanterol 62.5/25 $\mu \mathrm{g}$ OD was well tolerated, and the incidence of AEs and serious AEs was similar for combination therapy versus placebo and monocomponents. ${ }^{41,57}$ The rate of class-effect AEs associated with anticholinergic (eg, dry mouth) and BA (eg, tachycardia) agents was similar to that observed for placebo. ${ }^{41,57}$ In two 12-week studies, umeclidinium-vilanterol 62.5/25 $\mu \mathrm{g}$ and SFC 250/50 $\mu \mathrm{g}$ were both well tolerated and had similar AE profiles. ${ }^{27}$ In a pooled analysis of data from eight trials of umeclidinium-vilanterol 62.5/25 $\mu \mathrm{g}$ and 125/25 $\mu \mathrm{g}$, no increased risk of MACE was observed with active treatment versus placebo. ${ }^{58}$ Small numerical imbalances in cardiac ischemia were reported in some studies, but not others. As the imbalances were not dose-related, they were not considered drug-related. The incidence of cardiovascular AEs of special interest was comparable for umeclidinium-vilanterol, monocomponents, and placebo.

In the two 1-year tiotropium-olodaterol OD studies, the frequency of AEs was largely comparable between the combination- and individual component-treatment groups. The rates of MACE and cardiac events did not differ significantly between the combination and the individual component groups. ${ }^{30}$ Similarly, AE reporting (including MACE and Holter monitoring) in the two aclidiniumformoterol BID studies was generally comparable across all treatment groups. ${ }^{28,29}$

In a 2013 preliminary report from a retrospective cohort study of mortality in more than 5,000 patients with COPD, LAMA-LABA combination therapy reduced both all-cause (HR 0.53 [95\% CI 0.34-0.84]) and cardiovascular mortality (HR 0.39 [95\% CI 0.17-0.9]). ${ }^{59}$ Reductions in both mortality types were also observed with LAMA-LABA-inhaled corticosteroids, LABA-inhaled corticosteroids, and LAMAonly treatment.

\section{Discussion}

We identified 23 published Phase III RCTs of FDC bronchodilators in COPD. The data demonstrated that fixed-dose LAMA-LABA combinations significantly improved lung function compared with component monotherapies or single agents. . $23,30-32,35,36,39-41$ Indacaterolglycopyrronium OD, umeclidinium-vilanterol OD, and tiotropium-olodaterol OD also provided significant improvements over component monotherapies and/or

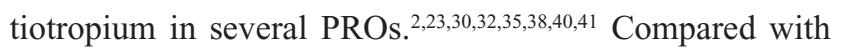
its components, aclidinium-formoterol BID improved symptoms (one study), ${ }^{28}$ but did not improve health status. ${ }^{28,29}$ Indacaterol-glycopyrronium and umeclidinium-vilanterol significantly improved lung function compared with SFC BID. ${ }^{26,27,39}$ Indacaterol-glycopyrronium also improved exacerbation rates in LANTERN and FLAME (Table 6), reduced dyspnea in ILLUMINATE, and led to reductions in use of rescue medication in ILLUMINATE and FLAME compared with SFC. ${ }^{24,26,27,39}$ The safety profiles of the FDC agents were similar to placebo and incidence of pneumonia significantly reduced with indacaterol-glycopyrronium versus SFC in FLAME. 2,23,26,30,32,36,39-41,54,56

Several studies have examined the relationship between improvements in lung function following LABA or LAMA monotherapy and improvements in other outcomes, 
such as SGRQ total score, TDI, exacerbation rate, and rescue-medication use. However, although significant or clinically relevant correlations appear at group levels, they tend to be only moderate, weak, or too weak to be useful at individual levels. ${ }^{16-19,21}$ This may be because some patients have very poor health despite only mild lung-function impairments or vice versa. ${ }^{17}$ Indeed, the health impact of COPD is not necessarily mediated entirely through expiratory airflow limitation; a better correlate may instead be exercise performance. ${ }^{17}$ The analyzed studies may also have been too short in duration to capture meaningful changes in exacerbations or health status, and only a few studies were available for some outcomes. ${ }^{18}$ Finally, the Hawthorne effect may also have played a role, as changes in $\mathrm{FEV}_{1}$ of 0 still resulted in a 2.5-point reduction in SGRQ score in some cases. ${ }^{18}$

Likewise, in trials of combination-bronchodilator therapy versus components, improvement in $\mathrm{FEV}_{1}$ was not always mirrored by improved PROs. For example, significant improvement in dyspnea for umeclidinium-vilanterol OD versus monocomponents occurred only for vilanterol (in one of three trials), despite improvements in $\mathrm{FEV}_{1} \cdot{ }^{23,41}$ Possible reasons for this include insufficient sensitivity/specificity in instruments assessing PROs. Additionally, such measurements as inspiratory capacity may be more strongly related to dyspnea and COPD pathophysiology than $\mathrm{FEV}_{1}{ }^{60}{ }^{6}$ Therefore, it may be useful to examine correlations between other outcomes instead, in larger sample sizes or longer-duration studies. Findings may still be somewhat limited though, as these end points are often only secondary, meaning power may be lacking.

Patient-selection criteria represent an important limitation of RCTs. Most trials recruit subjects from highly selective populations likely to represent less than 5\% of "real-life" patients. As such, the extrapolation of RCT data is limited. ${ }^{61}$ Populations are generally chosen to demonstrate the primary end point (usually lung function). Clinical trial participants tend to be less symptomatic than general patient populations, and clinical trials may exclude patients likely to benefit the most from treatment (as a maximum level of benefit may be reached sooner). Additionally, the most symptomatic patients in control arms may discontinue study treatment to obtain greater symptom relief. In contrast, real-life studies are likely to involve broader populations and treat each study arm to a similar level. Roche et al suggested a new framework to categorize the approach taken in clinical trials from highly controlled efficacy RCT management to usual clinical care. ${ }^{61}$ The positioning of studies on this scale can be useful as a descriptive classification. ${ }^{61}$
Future COPD trials may need to include more real-life patient populations and ecology of care. In addition, composite end points, such as lack of exacerbations and improved health status, may provide greater insight into the true benefits of treatment.

Additional studies of fixed-combination bronchodilators are needed to characterize further the relationship between $\mathrm{FEV}_{1}$ and PROs with these agents, as well as defining optimal strategies for their use in clinical practice. Should therapy be initiated with a single bronchodilator and then stepped up to a LABA-LAMA combination and/or triple therapy with LABA-LAMA plus another agent as needed, or should treatment commence with a LABA-LAMA in certain patients?

In conclusion, our review of a systematic literature search indicates that fixed-dose LABA-LAMA combinations significantly improved lung function compared with their component monotherapies. In general, LABA-LAMA combinations also improved other outcomes, including symptoms and health status, compared with the monotherapies, although some discrepancies between lung function and PROs were apparent. Further research is needed to explore the relationship between lung-function outcomes and PROs in patients receiving $\mathrm{LABA}-\mathrm{LAMA}$ combinations.

\section{Acknowledgments}

The authors were assisted in the preparation of the manuscript by Molly Heitz and Sarah Filcek, professional medical writers at CircleScience, an Ashfield company, part of UDG Healthcare PLC. Medical writing support was funded by Novartis Pharma AG (Basel, Switzerland).

\section{Author contributions}

All authors contributed to the concept and objectives of the review and provided guidance on the literature search, presentation, and discussion of the findings, as well as critically reviewing the article. In addition, all authors reviewed and approved the final manuscript.

\section{Disclosure}

AØ has received payment for lectures/speaking from Boehringer Ingelheim, GlaxoSmithKline, Meda, Sandoz, and Pfizer. He has advisory board membership with Boehringer Ingelheim, Novartis and Teva. DP has board membership with Aerocrine, Amgen, AstraZeneca, Boehringer Ingelheim, Chiesi, Meda, Mundipharma, Napp, Novartis, and Teva Pharmaceuticals; consultancy agreements with Almirall, Amgen, AstraZeneca, Boehringer Ingelheim, Chiesi, GlaxoSmithKline, Meda, Mundipharma, Napp, Novartis, Pfizer, Teva 
Pharmaceuticals, and Theravance; grants and unrestricted funding for investigator-initiated studies (conducted through Observational and Pragmatic Research Institute Pte Ltd) from UK National Health Service, British Lung Foundation, Aerocrine, AKL Ltd, AstraZeneca, Boehringer Ingelheim, Chiesi, Meda, Mundipharma, Napp, Novartis, Pfizer, Respiratory Effectiveness Group, Takeda, Teva Pharmaceuticals, Zentiva, and Theravance; payment for lectures/speaking engagements from Almirall, AstraZeneca, Boehringer Ingelheim, Chiesi, Cipla, GlaxoSmithKline, Kyorin, Meda, Merck, Mundipharma, Novartis, Pfizer, Skyepharma, Takeda, and Teva Pharmaceuticals; payment for manuscript preparation from Mundipharma and Teva Pharmaceuticals; payment for the development of educational materials from Novartis and Mundipharma; payment for travel/accommodation/meeting expenses from Aerocrine, Boehringer Ingelheim, Mundipharma, Napp, Novartis, Teva Pharmaceuticals, and AstraZeneca; funding for patient enrolment or completion of research from Chiesi, Teva Pharmaceuticals, Zentiva, and Novartis; stock/stock options from AKL Ltd which produces phytopharmaceuticals; owns $74 \%$ of the social enterprise Optimum Patient Care Ltd, UK and $74 \%$ of Observational and Pragmatic Research Institute Pte Ltd, Singapore; and is peer reviewer for grant committees of the Medical Research Council, Efficacy and Mechanism Evaluation programme, and HTA. Neither MT nor any member of his close family has any shares in pharmaceutical companies. In the last 3 years, he has received honoraria for speaking at sponsored meetings or satellite symposia at conferences from the following companies marketing respiratory and allergy products: Aerocrine, AstraZeneca, Boehringer Ingelheim, Novartis, GlaxoSmithKline and Teva. MT has received honoraria for attending advisory panels with Aerocrine, Almirall, AstraZeneca, Boehringer Ingelheim, Chiesi, GlaxoSmithKline, MSD, and Novartis. He has received sponsorship to attend international scientific meetings from AstraZeneca, GlaxoSmithKline, and Mundipharma; and has received funding for research projects from Almirall and GlaxoSmithKline. Neither TW nor his close family members have any shares in pharmaceutical companies. In the last 3 years, TW has received honoraria for speaking at sponsored meetings or satellite symposia at conferences from the following companies marketing respiratory and allergy products: Almirall, Astra Zeneca, Boehringer Ingelheim, Chiesi, Mundipharma, Novartis, GlaxoSmithKline, and Teva. He has received honoraria for attending advisory panels with Astra Zeneca, Boehringer Ingelheim, Chiesi, MSD, and Novartis, as well as receiving funding for research projects from Novartis. The authors report no other conflicts of interest in this work.

\section{References}

1. Global Initiative for Chronic Obstructive Lung Disease (GOLD). Global Strategy for the Diagnosis, Management, and Prevention of COPD. Bethesda (MD): GOLD; 2016. Available from: http://goldcopd. org/global-strategy-diagnosis-management-prevention-copd-2016/. Accessed October 23, 2015.

2. Bateman ED, Ferguson GT, Barnes N, et al. Dual bronchodilation with QVA149 versus single bronchodilator therapy: the SHINE study. Eur Respir J. 2013;42(6):1484-1494.

3. Cazzola M, Molimard M. The scientific rationale for combining long-acting $\beta_{2}$-agonists and muscarinic antagonists in COPD. Pulm Pharmacol Ther. 2010;23(4):257-267.

4. van Noord JA, Aumann JL, Janssens E, et al. Effects of tiotropium with and without formoterol on airflow obstruction and resting hyperinflation in patients with COPD. Chest. 2006;129(3):509-517.

5. Berton DC, Reis M, Siqueira AC, et al. Effects of tiotropium and formoterol on dynamic hyperinflation and exercise endurance in COPD. Respir Med. 2010;104(9):1288-1296.

6. Tashkin DP, Pearle J, Iezzoni D, Varghese ST. Formoterol and tiotropium compared with tiotropium alone for treatment of COPD. COPD. 2009;6(1):17-25.

7. Vogelmeier C, Kardos P, Harari S, Gans SJ, Stenglein S, Thirlwell J. Formoterol mono- and combination therapy with tiotropium in patients with COPD: a 6-month study. Respir Med. 2008;102(11):1511-1520.

8. Wang J, Jin D, Zuo P, Wang T, Xu Y, Xiong W. Comparison of tiotropium plus formoterol to tiotropium alone in stable chronic obstructive pulmonary disease: a meta-analysis. Respirology. 2011;16(2): 350-358.

9. Tashkin DP, Donohue JF, Mahler DA, et al. Effects of arformoterol twice daily, tiotropium once daily, and their combination in patients with COPD. Respir Med. 2009;103(4):516-524.

10. Tashkin DP, Littner M, Andrews CP, TomLinson L, Rinehart M, Denis-Mize K. Concomitant treatment with nebulized formoterol and tiotropium in subjects with COPD: a placebo-controlled trial. Respir Med. 2008;102(4):479-487.

11. Hanania NA, Boota A, Kerwin E, TomLinson L, Denis-Mize K. Efficacy and safety of nebulized formoterol as add-on therapy in COPD patients receiving maintenance tiotropium bromide: results from a 6-week, randomized, placebo-controlled, clinical trial. Drugs. 2009;69(9):1205-1216.

12. van Noord JA, Aumann JL, Janssens E, et al. Combining tiotropium and salmeterol in COPD: effects on airflow obstruction and symptoms. Respir Med. 2010;104(7):995-1004.

13. Jones PW, Agusti AG. Outcomes and markers in the assessment of chronic obstructive pulmonary disease. Eur Respir J. 2006;27(4): 822-832.

14. Jones PW, Beeh KM, Chapman KR, Decramer M, Mahler DA, Wedzicha JA. Minimal clinically important differences in pharmacological trials. Am J Respir Crit Care Med. 2014;189(3): 250-255.

15. Aisanov Z, Bai C, Bauerle O, et al. Primary care physician perceptions on the diagnosis and management of chronic obstructive pulmonary disease in diverse regions of the world. Int J Chron Obstruct Pulmon Dis. 2012;7:271-282.

16. Jones PW, Donohue JF, Nedelman J, Pascoe S, Pinault G, Lassen C. Correlating changes in lung function with patient outcomes in chronic obstructive pulmonary disease: a pooled analysis. Respir Res. 2011; $12: 161$.

17. Jones PW. Health status measurement in chronic obstructive pulmonary disease. Thorax. 2001;56(11):880-887.

18. Westwood M, Bourbeau J, Jones PW, Cerulli A, Capkun-Niggli G, Worthy G. Relationship between $\mathrm{FEV}_{1}$ change and patient-reported outcomes in randomised trials of inhaled bronchodilators for stable COPD: a systematic review. Respir Res. 2011;12:40.

19. Haruna A, Oga T, Muro S, et al. Relationship between peripheral airway function and patient-reported outcomes in COPD: a cross-sectional study. BMC Pulm Med. 2010;10:10. 
20. Watson L, Schouten JP, Löfdahl CG, Pride NB, Laitinen LA, Postma DS. Predictors of COPD symptoms: does the sex of the patient matter? Eur Respir J. 2006;28(2):311-318.

21. Donohue JF, Jones P, Bartels C, et al. Relationship between change in trough $\mathrm{FEV}_{1}$ and COPD patient outcomes: pooled analysis of 23 clinical trials in patients with COPD. Eur Respir J. 2015;46 Suppl 59: PA1013.

22. Moher D, Liberati A, Tetzlaff J, Altman DG. Preferred reporting items for systematic reviews and meta-analyses: the PRISMA statement. PLoS Med. 2009;6(7):e1000097.

23. Decramer M, Anzueto A, Kerwin E, et al. Efficacy and safety of umeclidinium plus vilanterol versus tiotropium, vilanterol, or umeclidinium monotherapies over 24 weeks in patients with chronic obstructive pulmonary disease: results from two multicentre, blinded, randomised controlled trials. Lancet Respir Med. 2014;2(6):472-486.

24. Zhong N, Wang C, Zhou X, et al. LANTERN: a randomized study of QVA149 versus salmeterol/fluticasone combination in patients with COPD. Int J Chron Obstruct Pulmon Dis. 2015;10:1015-1026.

25. Buhl R, Gessner C, Schuermann W, et al. Efficacy and safety of oncedaily QVA149 compared with the free combination of once-daily tiotropium plus twice-daily formoterol in patients with moderate-to severe COPD (QUANTIFY): a randomised, non-inferiority study. Thorax. 2015;70(4):311-319.

26. Wedzicha JA, Banerji D, Chapman KR, et al. Indacaterolglycopyrronium versus salmeterol-fluticasone for COPD. N Engl J Med. 2016;374(23):2222-2234.

27. Donohue JF, Worsley S, Zhu CQ, Hardaker L, Church A. Improvements in lung function with umeclidinium/vilanterol versus fluticasone propionate/salmeterol in patients with moderate-to-severe COPD and infrequent exacerbations. Respir Med. 2015;109(7):870-881.

28. Singh D, Jones PW, Bateman ED, et al. Efficacy and safety of aclidinium bromide/formoterol fumarate fixed-dose combinations compared with individual components and placebo in patients with COPD (ACLIFORM-COPD): a multicentre, randomised study. BMC Pulm Med. 2014;14:178.

29. D'Urzo AD, Rennard SI, Kerwin EM, Mergel V, Leslbaum AR, Caracta CF. Efficacy and safety of fixed-dose combinations of aclidinium bromide/formoterol fumarate: the 24-week, randomized, placebocontrolled AUGMENT COPD study. Respir Res. 2014;15:123.

30. Buhl R, Maltais F, Abrahams R, et al. Tiotropium and olodaterol fixeddose combination versus mono-components in COPD (GOLD 2-4). Eur Respir J. 2015;45(4):969-979.

31. Beeh KM, Westerman J, Kirsten AM, et al. The 24-h lung-function profile of once-daily tiotropium and olodaterol fixed-dose combination in chronic obstructive pulmonary disease. Pulm Pharmacol Ther. 2015;32:53-59.

32. Maleki-Yazdi MR, Kaelin T, Richard N, Zvarich M, Church A. Efficacy and safety of umeclidinium/vilanterol $62.5 / 25 \mathrm{mcg}$ and tiotropium $18 \mathrm{mcg}$ in chronic obstructive pulmonary disease: Results of a 24-week, randomized, controlled trial. Respir Med. 2014;108(12): $1752-1760$

33. Maltais F, Singh S, Donald AC, et al. Effects of a combination of umeclidinium/vilanterol on exercise endurance in patients with chronic obstructive pulmonary disease: two randomized, double-blind clinical trials. Ther Adv Respir Dis. 2014;8(6):169-181.

34. O'Donnell D, Casaburi R, De Sousa D, et al. Effects of 6 weeks' treatment with once-daily tiotropium and olodaterol fixed-dose combination on inspiratory capacity and exercise endurance in patients with COPD: the MORACTO studies. Am J Respir Crit Care Med. 2015; 191(1):A3972.

35. Beeh KM, Korn S, Beier J, et al. Effect of QVA149 on lung volumes and exercise tolerance in COPD patients: the BRIGHT study. Respir Med. 2014;108(4):584-592.

36. Dahl R, Chapman KR, Rudolf M, et al. Safety and efficacy of dual bronchodilation with QVA149 in COPD patients: the ENLIGHTEN study. Respir Med. 2013;107(10):1558-1567.
37. Dahl R, Jadayel D, Alagappan VK, Chen H, Banerji D. Efficacy and safety of QVA149 compared to the concurrent administration of its monocomponents indacaterol and glycopyrronium: the BEACON study. Int J Chron Obstruct Pulmon Dis. 2013;8:501-508.

38. Mahler DA, Decramer M, D'Urzo A, et al. Dual bronchodilation with QVA149 reduces patient-reported dyspnoea in COPD: BLAZE study. Eur Respir J. 2014;43(6):1599-1609.

39. Vogelmeier CF, Bateman ED, Pallante J, et al. Efficacy and safety of once-daily QVA149 compared with twice-daily salmeterol-fluticasone in patients with chronic obstructive pulmonary disease (ILLUMINATE): a randomised, double-blind, parallel group study. Lancet Respir Med. 2013;1(1):51-60.

40. Wedzicha JA, Decramer M, Ficker JH, et al. Analysis of chronic obstructive pulmonary disease exacerbations with the dual bronchodilator QVA149 compared with glycopyrronium and tiotropium (SPARK): a randomised, double-blind, parallel-group study. Lancet Respir Med. 2013;1(3):199-209.

41. Donohue JF, Maleki-Yazdi MR, Kilbride S, Mehta R, Kalberg C, Church A. Efficacy and safety of once-daily umeclidinium/vilanterol $62.5 / 25 \mathrm{mg}$ in COPD. Respir Med. 2013;107(10):1538-1546.

42. Donohue JF, Singh D, Munzu C, Kilbride S, Church A. Magnitude of umeclidinium/vilanterol lung function effect depends on monotherapy responses: results from two randomised controlled trials. Respir Med. 2016;112:65-74.

43. Singh D, Worsley S, Zhu CQ, Hardaker L, Church A. Umeclidinium/ vilanterol versus fluticasone propionate/salmeterol in COPD: a randomised trial. BMC Pulm Med. 2015;15:91.

44. Donohue JF, Niewoehner D, Brooks J, O'Dell D, Church A. Safety and tolerability of once-daily umeclidinium/vilanterol $125 / 25 \mathrm{mcg}$ and umeclidinium $125 \mathrm{mcg}$ in patients with chronic obstructive pulmonary disease: results from a 52-week, randomized, double-blind, placebocontrolled study. Respir Res. 2014;15:78.

45. Maltais F, Iturra JB, Kirsten A, et al. Effects of 12 weeks of once-daily tiotropium and olodaterol fixed-dose combination on exercise endurance in patients with COPD. Eur Respir J. 2014;44 Suppl 58:P283.

46. Donohue JF, Soong W, Wu X, Shrestha P, Lei A. Long-term safety of aclidinium bromide/formoterol fumarate fixed-dose combination: results of a randomized 1-year trial in patients with COPD. Respir Med. 2016;116:41-48.

47. Vogelmeier C, Paggiaro PL, Dorca J, et al. Efficacy and safety of aclidinium/formoterol versus salmeterol/fluticasone: a phase 3 COPD study. Eur Respir J. 2016;48(4):1030-1039.

48. Reisner C, Rose E, Strom S. Fixed combination of glycopyrrolate and formoterol MDI (GFF-MDI) demonstrates superior inspiratory capacity (IC) compared to tiotropium DPI (Tio) following 7 days dosing, in a randomized, double-blind, placebo-controlled phase 2B study in patients with COPD. Eur Respir J. 2011;38 Suppl 55:P879.

49. Reisner C, Rennard S, Fogarty C, et al. Pearl Therapeutics' combination LAMA/LABA MDI (GFF-MDI, PT003) provides a significant benefit on home peak expiratory flow rate (PEFR) and reduces the need for rescue albuterol use compared to its components administered alone, Spiriva Handihaler, and Foradil Aerolizer in a randomized, doubleblind, placebo-controlled phase 2B study in patients with COPD. Poster presented at: American Thoracic Society (ATS) International Conference; May 18-23, 2012; San Francisco, CA.

50. Reisner C, Gotfried M, Denenberg MB, et al. Low doses of Pearl Therapeutics' LAMA/LABA combination MDI (GFF MDI, PT003) provide superior bronchodilation compared to components and to open-label Spiriva HandiHaler in a randomized, double-blind, placebocontrolled Phase IIB study in patients with COPD. Poster presented at: American Thoracic Society (ATS) International Conference; May 17-22, 2013; Philadelphia, PA.

51. Chapman KR, Bateman ED, Olsson P, Chen H, Banerji D, Fogel R. A significant proportion of patients with COPD show marked improvements in lung function with QVA149 (high responders): a post-hoc analysis of the SHINE study. Chronic Obstr Pulm Dis. 2015;2(2):159. 
52. Bateman ED, Vogelmeier C, Chen H, Banerji D. Comparison of COPD exacerbations with once-daily QVA149 versus twice-daily salmeterol/fluticasone combination: the ILLUMINATE study. Chest. 2014;145(3):409A.

53. Jones PW. St. George's Respiratory Questionnaire: MCID. COPD. 2005; 2(1):75-79.

54. Wedzicha JA, Dahl R, Buhl R, et al. Pooled safety analysis of the fixeddose combination of indacaterol and glycopyrronium (QVA149), its monocomponents, and tiotropium versus placebo in COPD patients. Respir Med. 2014;108(10):1498-1507.

55. Ferguson G, Barnes N, Mehta R, D'Andrea P, Chen H, Banerji D. Cardio- and cerebro-vascular safety profile of QVA149 in patients with COPD: a pooled analysis. Am J Respir Crit Care Med. 2013; 187(1):A1488.

56. Van de Maele B, Fabbri LM, Martin C, Horton R, Dolker M, Overend T. Cardiovascular safety of QVA149, a combination of indacaterol and NVA237, in COPD patients. COPD. 2010;7(6):418-427.

57. US Food and Drug Administration. NDA 203-975: umeclidinium and vilanterol inhalation powder for the long-term, once-daily maintenance treatment of airflow obstruction in patients with chronic obstructive pulmonary disease (COPD). 2013. Available from: http://www.fda.gov/ downloads/advisorycommittees/committeesmeetingmaterials/drugs/ pulmonary-allergydrugsadvisorycommittee/ucm367411.pdf. Accessed October 23, 2015.
58. Naccarelli G, Finkle J, Chopra B, Brooks J, Harris S, Church A. Cardiovascular safety of umeclidinium/vilanterol in COPD: results from eight randomized clinical trials. Poster presented at: American Thoracic Society (ATS) International Conference; May 16-21, 2014; San Diego, CA.

59. Manoharan A, Short PM, Anderson WJ, Lipworth BJ. Impact of long-acting bronchodilator therapy on mortality in COPD: a real-life retrospective cohort study. Thorax. 2013;68 (Suppl 3):A179-A180.

60. Di Marco F, Milic-Emili J, Boveri B, et al. Effect of inhaled bronchodilators on inspiratory capacity and dyspnoea at rest in COPD. Eur Respir J. 2003;21(1):86-94.

61. Roche N, Reddel HK, Agusti A, et al. Integrating real-life studies in the global therapeutic research framework. Lancet Respir Med. 2013; 1(10):e29-e30.

62. Asai K, Minakata Y, Hirata K, et al. QVA149 once-daily is safe and well tolerated and improves lung function and health status in Japanese patients with COPD: the ARISE study. Eur Respir J. 2013; 42 Suppl 57:694s.

63. D'Urzo A, Rennard S, Kerwin E, et al. One-year efficacy of aclidinium/ formoterol fixed-dose combination in COPD patients: the AUGMENT COPD study. Poster presented at: European Respiratory Society (ERS) International Congress; September 6-10, 2014; Munich. 


\section{Supplementary materials}

Table SI Search strategy and results for published manuscripts and congress abstracts

\begin{tabular}{|c|c|c|}
\hline Search number & Search terms & Number of records \\
\hline SI & $\begin{array}{l}\text { MeSH.EXACT.EXPLODE (“Bronchodilator Agents") AND MeSH.EXACT.EXPLODE } \\
\text { ("Drug Combinations”) }\end{array}$ & $821^{a}$ \\
\hline S2 & $\begin{array}{l}\text { "Fixed-dose combination" OR "Fixed dose combination" OR "Fixed-dose long-acting } \\
\text { combination" OR "Fixed dose long-acting combination" OR "Fixed-dose combinations" } \\
\text { OR "Fixed dose combinations" OR "Fixed-dose long-acting combinations" OR "Fixed dose } \\
\text { long-acting combinations" OR "fixed combination" OR "fixed combinations" OR "LABA/ } \\
\text { LAMA" OR "LAMA/LABA" OR "dual bronchodilator" OR "dual bronchodilators" OR } \\
\text { "dual bronchodilation" OR "dual-acting bronchodilator" OR "dual-acting bronchodilators" } \\
\text { OR "dual-acting bronchodilation" OR "QVAI49" OR "QVA-I49" OR "QVA I49" } \\
\text { OR "glycopyrronium/indacaterol" OR "indacaterol/glycopyrronium" OR "Anoro" OR } \\
\text { "umeclidinium/vilanterol" OR Embase.EXACT ("glycopyrronium bromide plus indacaterol") }\end{array}$ & $6,959^{b}$ \\
\hline S3 & $\begin{array}{l}\text { MeSH.EXACT.EXPLODE (“Pulmonary Disease, Chronic Obstructive”) OR "chronic } \\
\text { obstructive pulmonary disease" OR "COPD” OR "Chronic Obstructive Lung Disease" OR } \\
\text { "Chronic Obstructive Airway Disease" }\end{array}$ & $90,402^{\mathrm{b}}$ \\
\hline S4 & $(\mathrm{SI}$ OR S2) AND S3 & $444^{\mathrm{a}}$ \\
\hline
\end{tabular}

Notes: aDuplicate citations removed from result count; ${ }^{b}$ result count includes duplicate citations. ProQuest search, including Biosis, Biosis previews, Embase, and Medline databases. Searches were limited to publications from January I, 2006 to July 3I, 2014 and English-language articles.

Abbreviations: EXACT, EXAcerbations of COPD Tool; EXPLODE, terms indexed as subterms included; LABA, long-acting $\beta_{2}$-agonist; LAMA, long-acting muscarinic antagonist; MeSH, Medical Subject Headings.

Table S2 Congress abstract search strategy and results

\begin{tabular}{ll}
\hline Congress abstracts searched & - Annual Congress of the European Respiratory Society \\
- Annual International Conference of the American Thoracic Society \\
- Annual Winter Meeting of the British Thoracic Society \\
- Biennial International Multidisciplinary Conference on Chronic Obstructive Pulmonary Disease \\
- Biennial World Conference of the International Primary Care Respiratory Group \\
- CHEST \\
- Annual Congress of the Asian Pacific Society of Respirology \\
"Fixed-dose combination" OR "Fixed dose combination" OR "Fixed-dose long-acting combination" OR "Fixed dose \\
long-acting combination" OR "Fixed-dose combinations" OR "Fixed dose combinations" OR "Fixed-dose long-acting \\
combinations" OR "Fixed dose long-acting combinations" OR "fixed combination" OR "fixed combinations" OR \\
"LABA/LAMA" OR "LAMA/LABA" OR "dual bronchodilator" OR "dual-bronchodilator" OR "dual-bronchodilators" \\
OR "dual-bronchodilation" OR "dual bronchodilators" OR "dual bronchodilation" OR "dual-acting bronchodilator" \\
OR "dual-acting bronchodilators" OR "dual-acting bronchodilation" OR "dual acting bronchodilator" OR "dual \\
acting bronchodilators" OR "dual acting bronchodilation" OR "QVAI49" OR "QVA-I49" OR "QVA I49" OR \\
"glycopyrronium/indacaterol" OR "indacaterol/glycopyrronium" OR "Anoro" OR "umeclidinium/vilanterol" OR \\
"glycopyrronium bromide plus indacaterol" OR "glycopyrronium plus indacaterol" \\
285
\end{tabular}

Note: Available abstracts from January I, 2009 to May 20, 2015 were included in the literature search.

International Journal of COPD

\section{Publish your work in this journal}

The International Journal of COPD is an international, peer-reviewed journal of therapeutics and pharmacology focusing on concise rapid reporting of clinical studies and reviews in COPD. Special focus is given to the pathophysiological processes underlying the disease, intervention programs, patient focused education, and self management protocols.

\section{Dovepress}

This journal is indexed on PubMed Central, MedLine and CAS. The manuscript management system is completely online and includes a very quick and fair peer-review system, which is all easy to use. Visit $\mathrm{http}: / /$ www.dovepress.com/testimonials.php to read real quotes from published authors. 